UTTG-14-02

HUTP-02/A063

December 9, 2002

hep-th/0212098

\title{
Quantum Deconstruction of a 5D SYM and its Moduli Space*
}

\author{
Amer Iqbal $^{(1)}$ and Vadim S. Kaplunovsky ${ }^{(2)}$ \\ (1) Jefferson Laboratory \\ Harvard University, \\ Cambridge, MA 02138, USA \\ iqbal@fresnel.harvard.edu \\ (2) Theory Group, Department of Physics \\ University of Texas at Austin, \\ Austin, TX 78712, USA \\ vadim@physics.utexas.edu
}

\begin{abstract}
We deconstruct the fifth dimension of the 5D SYM theory with $S U(M)$ gauge symmetry and Chern-Simons level $k_{\mathrm{cs}}=M$ and show how the 5D moduli space follows from the non-perturbative analysis of the $4 \mathrm{D}$ quiver theory. The $5 \mathrm{D}$ coupling $h=1 / g_{5}^{2}$ of the un-broken $S U(M)$ is allowed to take any non-negative values, but it cannot be continued to $h<0$ and there are no transitions to other phases of the theory. The alternative UV completions of the same 5D SYM - via M theory on the $\mathbb{C}^{3} / \mathbb{Z}_{2 M}$ orbifold or via the dual five-brane web in type IIB string theory - have identical moduli spaces: $h \geq 0$ only, and no flop transitions. We claim these are intrinsic properties of the $S U(M)$ SYM theory with $k_{\mathrm{cs}}=M$.
\end{abstract}

* Research supported by the US National Science Foundation (grants NSF-DMS/00-74329 and PHY0071512), by the Robert A. Welsh foundation, and by the US-Israel Bi-National Science Foundation. 


\section{Introduction}

In the course of developing the string/M theory over the last generation, we have learned much about the ordinary quantum field theories in various spacetime dimensions. This is particularly true in $D>4$ where interactive field theories are non-renormalizable and therefore ill-defined at the quantum level — but embedding into a string or M theory provides an ultraviolet completion which respects all the important symmetries of the QFT such as SUSY, gauge and Lorentz invariance [1, 2, 3, 4, 5]. For example, a 5D gauge theory with $\mathcal{N}=1$ SUSY can be UV completed as: (1) type I' string theory on D4-brane probes in a background of D8-branes and O8 orientifold planes [4], or (2) M theory compactified on a singular Calabi-Yau threefolds [6, 7, 8], or (2) type IIB string theory on a web of five-branes [9, 10, 11]. With the help of 8 unbroken supercharges, these UV completions allow us to calculate the exact moduli-dependent gauge couplings of the 5D theory and the global geometry of its moduli space, including 'flop' transitions to different 5D phases [12], sometimes involving strongly-coupled sectors with non-trivial IR fixed points.

Unfortunately, using a 'stringy' UV completion of a 5D supersymmetric gauge theory to derive its phase structure poses a troubling question: Is this phase structure an inherent property of the $5 D$ theory (regardless of a $U V$ regulator), or is it an artifact of string/M theory? To resolve this quandary, we need to compare the phase structures of different UV completions of the same 5D theory. However, there is no use in comparing the stringy UV completions to each other: They are dual as string/M theories, and the agreement between their 5D phase structures does not prove anything besides confirming the duality. Instead, the stringy completions should be compared to a completely different, non-stringy UV completion of the same 5D theory. And of course, to study the 5D phase structures, we need this non-stringy completion to work even when the 5D couplings are strong [12].

In this article, we use dimensional deconstruction [13] of the fifth spacetime dimension as a non-stringy UV regulator of a quantum 5D SYM theory. That is, we replace the continuous $x^{4}$ coordinate with a discrete lattice and re-interpret the $5 \mathrm{D}$ gauge symmetry $S U(M)$ as a $4 \mathrm{D}$ gauge symmetry $[S U(M)]^{N}$ — one $S U(M)$ factor for each 4 D layer $x^{4}=\ell a(\ell=1,2, \ldots, N)$. In the process, we break 4 out of 8 supersymmetries of the 5D theory and reduce its Lorentz 
symmetry down to $S O(1,3)$, but the broken symmetries re-appear in the continuum limit of distances much larger than the lattice spacing $a$. In the opposite limit of short distances, the deconstructed theory is effectively four-dimensional, renormalizable and asymptotically free; this makes deconstruction a good UV regulator even when the low-energy couplings are strong. Furthermore, at all energies the deconstructed theory has 4 unbroken supercharges, which allows for exact calculations of holomorphic quantities, such as moduli-dependent abelian gauge couplings of the Coulomb branch of the theory.

Our main results are (1) the method for converting the exact non-perturbative features of the deconstructed theories into the exact moduli spaces and phase structures of the 5D continuum limit, and (2) the fact that these moduli spaces and phase structures are exactly as for the stringy UV completions of the same 5D theory. Consequently, we believe that the phase structure is inherent in the $5 \mathrm{D}$ theory and does not depends on the manner of its UV completion.

For simplicity, the analysis of this article is limited to the 5D SYM theories with $S U(M)$ gauge symmetries and no hypermultiplets (i.e., the flavorless $\mathrm{SQCD}_{5}$ ). Furthermore, we use the simplest 4D quiver to implement the latticized fifth dimension, namely a single $S U(M)_{\ell}$ vector multiplet for each lattice node $\ell$ and a single bi-fundamental chiral multiplet $\left(\mathbf{M}_{\ell}, \overline{\mathbf{M}}_{\ell+1}\right)$ for each lattice link, without any additional 4D fields. Consequently, in the $5 \mathrm{D}$ continuum limit, the SYM theory has a specific Chern-Simons level, namely $k_{\mathrm{cs}}=M$. Changing the 5D Chern-Simons level or/and adding the flavors involves more complicated quivers, and we shall present them in a separate follow-up article [14].

But before we delve into the details of deconstruction, etc., let us briefly review the basic features of the parameter/moduli spaces of the 5D SYM theories. First of all, we need to distinguish between the non-dynamical parameters of a 5D theory and the dynamical moduli of its exactly degenerate vacua. In $5 \mathrm{D}$, the scalar fields of an $\mathcal{N}=1 \mathrm{SYM}$ theory form a single real adjoint multiplet of the gauge symmetry $G$ and there is no scalar potential. Consequently, all the vacua of the theory belong to the Coulomb branch where $G$ is generically broken to its Cartan subgroup $U(1)^{r}$ and the space of dynamical moduli scalars is simply $\mathbb{R}^{r}$ divided by the Weyl group of $G$. For example, $G=S U(2)$ has rank 1 and Weyl symmetry $\mathbb{Z}_{2}$, hence the dynamical moduli space $\mathbb{R} / \mathbb{Z}_{2}=\mathbb{R}^{+}$, or in other words there is a single real 
modulus $\phi \geq 0$. More generally, $G=S U(M)$ has rank $r=M-1$ and Weyl symmetry $S_{M}$, hence the $\mathbb{R}^{M-1} / S_{M}$ moduli space. Indeed, the dynamical moduli of the $S U(M)$ theory are the $M$ eigenvalues $\left(\phi_{1}, \phi_{2}, \ldots, \phi_{M}\right)$ of an hermitian traceless $M \times M$ matrix, but they are subject to a linear constraint

$$
\phi_{1}+\phi_{2}+\cdots+\phi_{M}=0
$$

and the Weyl symmetry of arbitrary permutations of $\phi_{i}$. In other words, the moduli space spans sets of $M$ ordered eigenvalues satisfying

$$
\phi_{1} \leq \phi_{2} \leq \cdots \leq \phi_{M}
$$

in addition to (11). Generically, all the inequalities (8) are strict and the $S U(M)$ is completely broken down to $U(1)^{M-1}$; an equality $\phi_{i}=\phi_{i+1}=\cdots=\phi_{i+k}$ signals a surviving non-abelian subgroup $S U(k+1) \subset S U(M)$.

The metric for the dynamical moduli scalars follows from a cubic prepotential $\mathcal{F}(\phi)$, which also governs the gauge couplings and the Chern-Simons couplings of the gauge fields:

$$
\begin{aligned}
\mathcal{L}_{\text {bosonic }}= & \frac{1}{8 \pi^{2}} \frac{\partial^{2} \mathcal{F}}{\partial \phi_{i} \partial \phi_{j}}\left[\frac{1}{2} D_{\mu} \phi^{i} D^{\mu} \phi^{j}+\frac{1}{4} F_{\mu \nu}^{i} F^{i, \mu \nu}\right] \\
& +\frac{1}{96 \pi^{2}} \frac{\partial^{3} \mathcal{F}}{\partial \phi_{i} \partial \phi_{j} \partial \phi_{k}} \epsilon^{\alpha \beta \gamma \mu \nu}\left[A_{\alpha}^{i} F_{\beta \gamma}^{j} F_{\mu \nu}^{k}+\begin{array}{c}
\text { non-abelian terms, } \\
\text { if appropriate }
\end{array}\right] .
\end{aligned}
$$

The Chern-Simons couplings have quantized coefficients, and for any gauge-invariant UV completion of the theory these coefficients are completely determined at the one-loop level of the perturbation theory; there are no higher-order perturbative or non-perturbative corrections. Consequently, the prepotential and the gauge couplings are also completely determined at the one-loop level [4]. For the Coulomb branch of the flavorless $S U(M)$, we have

$$
\mathcal{F}^{S U(M)}(\phi)=\frac{h}{2} \sum_{i} \phi_{i}^{2}+\frac{k_{C S}}{6} \sum_{i} \phi_{i}^{3}+\frac{1}{6} \sum_{i>j}\left|\phi_{i}-\phi_{j}\right|^{3}
$$

and hence

$$
\left[\frac{8 \pi^{2}}{g^{2}}\right]_{i j}=\frac{\partial^{2} \mathcal{F}^{S U(M)}}{\partial \phi_{i} \partial \phi_{j}}=\left[h+k_{C S} \phi_{i}+\sum_{k}\left|\phi_{i}-\phi_{k}\right|\right] \times \delta_{i j}-\left|\phi_{i}-\phi_{j}\right|
$$

Here $h$ is the tree-level inverse gauge coupling of the unbroken $S U(M)$ theory and $k_{\mathrm{cs}}$ is its 
tree-level Chern-Simons coefficient. From the $S U(M)$ point of view, $h$ is a non-dynamical constant parameter, but it can be promoted to a full vector supermultiplet of a larger theory. Consequently, we have a combined parameter/moduli space spanned by the $h$ and all the $\phi_{i}$, and it is the geometry of this combined parameter/moduli space which is the real question here. Specifically, we want to know whether $h$ can take negative values, and if yes, we want to learn everything about the $h<0$ domain of the parameter/moduli space, such as the allowed ranges of the $\phi_{i}$ moduli for $h<0$, the details of the flop transition between the $h>0$ and $h<0$ domains, and most importantly, the physics of the $h<0$ phase of the theory.

The answers to these questions depend on the Chern-Simons level of the SYM theory in question. The theory with $k_{\mathrm{cs}}=M$ (or equivalently $k_{\mathrm{cs}}=-M$ ) - the main subject of this article - does not have any $h<0$ phases; instead, the parameter space is limited to $h \geq 0$ and there are no flop transitions. On the other hand, for $\left|k_{\mathrm{cs}}\right|<M$ the parameter space has both $h>0$ and $h<0$ domains connected via a flop transition at the infinite-coupling point $h=0$. Physically, the $h<0$ phase has a not-trivial IR fixed point and its low-energy limit is governed by a $5 \mathrm{D}$ superconformal field theory rather than SYM.

Deriving these phase structures involves non-perturbative analysis of the strongly coupled 5D SYM - or rather of its UV completion. We claim however that different UV completions of the same 5D SYM have identical phase structures, which are therefore inherent properties of the 5D theory itself, regardless of the completion. In this article, we verify this claim for the $k_{\mathrm{cs}}=M$ theory by comparing its UV completions via $\mathrm{M} /$ string theory with the completion via dimensional deconstruction. The theories with $\left|k_{\mathrm{cs}}\right|<M$ will be addressed in detail in the follow-up article [14].

The rest of this article is organized as follows: In the following section 2, we study the stringy UV completions of the 5D SYM theories. In $\S 2.1$, we put M theory on a CY orbifold singularity $\mathbb{C}^{3} / \mathbb{Z}_{2 M}$ and show that a partial resolution of this singularity gives rise to a flavorless $S U(M)$ in $5 \mathrm{D}$. The moduli/parameter space of the $5 \mathrm{D}$ Coulomb branch follows from the intersections of the 4-cycles of the completely resolved singularity, and we show that this orbifold model has no flop transitions and that its prepotential corresponds to $k_{\mathrm{cs}}=M$. In $\S 2.2$ we construct a dual type IIB string model in which the 5D physics arises from a web 
of $(p, q)$ five-branes [9]; the $S U(M)$ gauge symmetry follows from $M$ parallel five-branes of the same type. For $k_{\mathrm{cs}}= \pm M$, the web has a unique topology with 2 parallel external legs separated by the distance $h$; consequently, the 5D SYM has no flop transitions and only the $h \geq 0$ values are allowed. But for $\left|k_{\mathrm{cs}}\right|<M$, none of the external legs are parallel, and their relative motion allows both positive and negative values of the $h$ parameter.

In section 3 we focus on the dimensional deconstruction. After a brief review of deconstruction at the semi-classical level, we consider the quantum effects in the $4 \mathrm{D}[S U(M)]^{N}$ quiver theory. In $\S 3.1$, we use holomorphy (due to 4 unbroken supercharges of the deconstructed theory) to construct the moduli space of the Coulomb branch of the quantum quiver and the Seiberg-Witten curve encoding the moduli dependence of the gauge couplings. In the large quiver limit $N \rightarrow \infty$ we recover the un-compactified 5D theory, and we show that the moduli dependence of its gauge couplings is exactly as in eq. (5) for $k_{\mathrm{cs}}=M$. Furthermore, the quantum corrections to the quiver's moduli space translate into $5 \mathrm{D}$ terms as $h \geq 0$, and indeed there are no flop transitions in the large $N$ limit of this quiver. In $\S 3.2$, we deconstruct the 5D Chern-Simons coupling of the un-broken $S U(M)$ theory directly from the quiver, without any help from SUSY. We show that the quiver theory has Wess-Zumino couplings with coefficients $k_{\mathrm{wz}}=M$ for every bi-linear scalar field of the quiver, which deconstruct the 5D Chern-Simons couplings at the level $k_{\mathrm{cs}}=k_{\mathrm{wz}}=M$. This confirms the 5D identity of the deconstructed theory, and it also tells us we need more complicated quivers to deconstruct the $5 \mathrm{D}$ theories with $k_{\mathrm{cs}} \neq \pm M$.

Finally, in the Appendix we calculate the abelian gauge couplings from the hyperelliptic Seiberg-Witten curve of the 4D quiver constructed in $\S 3.1$. 


\section{$2 \quad$ M Theory Orbifolds and $(p, q)$ Five-Brane Webs}

In this section we discuss the string/M-theory based UV completions of the 5D SYM theories. First, we compactify M Theory on singular Calabi-Yau threefolds and show that a particular $\mathbb{C}^{3} / \mathbb{Z}_{2 M}$ orbifold singularity (or rather its partial blowup) gives rise to the flavorless $S U(M)$ SYM in five dimensions [12]. The parameter space of this model had no flop transitions, and the Chern-Simons level of this model turns out to be $k_{\mathrm{cs}}=M$.

In the second half of this section, we take a dual IIB string theory embedding in which the $5 \mathrm{D}$ physics arises from a web of $(p, q)$ five-branes [9]. The web diagram dual to the $\mathbb{C}^{3} / \mathbb{Z}_{2 M}$ orbifold has $M$ parallel internal lines — which explains the $S U(M)$ gauge symmetry — and two parallel external legs — which explains the $k_{\mathrm{cs}}=M$ and the absence of flop transitions.

\subsection{Theory on the $\mathbb{C}^{3} / \mathbb{Z}_{2 M}$ Orbifold}

M theory compactified on a smooth Calabi-Yau threefold gives rise to an abelian gauge theory in the five un-compactified dimensions. The 5D gauge fields $A_{\mu}^{i}$ follow from the 11D 3-form $C_{\lambda \mu \nu}$ reduced on the $H^{(1,1)}$ cohomology of the threefold,

$$
C=\sum_{i=1}^{h_{1,1}} A^{i} \wedge \omega^{i}, \quad \omega^{i} \in H^{(1,1)}(\mathrm{CY}) .
$$

To get a non-abelian 5D gauge symmetry we need a singularity in which a complex surface $\mathbb{S}$ collapses to a complex curve $B[12, \mathbb{8}$. In this collapse, the 2 -cycles contained in $\mathbb{S}$ and fibered over the base $B$ shrink to zero area, and the M2-branes and anti-M2-branes wrapped around these shrinking cycles give rise to the massless non-abelian vector multiplets. The rank $r$ of the non-abelian symmetry is given by the number of homologically-independent 4cycles $\left(S_{1}, \ldots, S_{r}\right)$ involved in the collapsing surface $\mathbb{S}$ while the Dynkin diagram depends on the intersection matrix of the shrinking 2-cycles fibered over the whole base. The exceptional fibers - if any — give rise to the matter fields, $i$. e. the charged hypermultiplets [8].

The inverse gauge coupling $h=\left(8 \pi^{2} / g^{2}\right)$ of the $5 \mathrm{D}$ theory is proportional to the area of the base cycle $B$. When the base area shrinks to zero as well, the theory becomes infinitely 
strongly coupled and its infrared limit becomes superconformal. For some singularities, the base cannot be un-shrunk apart from the fibers and the 4 -cycle family $\left(S_{1}, \ldots, S_{r}\right)$ collapse to a single point; such singularities give rise to exotic 5D superconformal theories which do not follow from $g=\infty$ gauge theories. The best known example of such exotic SCFT is the $E_{0}$ of rank $r=1$ [7], 6].

The simplest example of a singularity where a complex surface collapses to a point is an orbifold point $\mathbb{C}^{3} / \mathbb{Z}_{N}$. The orbifolding symmetry $\mathbb{Z}_{N}$ acts according to

$$
\mathbb{Z}_{N}:\left(z_{1}, z_{2}, z_{3}\right) \mapsto\left(e^{2 \pi i a / N} z_{1}, e^{2 \pi i b / N} z_{2}, e^{2 \pi i c / N} z_{3}\right), \quad a+b+c=N
$$

and the fixed point $(0,0,0)$ hides a blown-down weighted projective space $\mathbb{W P}^{2}$ with weights proportional $(a, b, c)$ modulo $N$. Often, this $\mathbb{W P}^{2}$ is itself singular, hence a complete resolution involves $r>1$ homologically-independent 4-cycles, and when these cycles re-collapse to a line rather than a point, one obtains a $5 \mathrm{D}$ gauge theory of a non-abelian rank $r>1$.

Without loss of generality we assume $a=1$, hence the collapsed surface is

$$
\mathbb{S}=\mathbb{W} \mathbb{P}^{2}[1, b, c]
$$

whose toric diagram is a simple triangle with vertices $(1,0),(0,1)$ and $(-c,-b)$; for example, the diagram for $\mathbb{W P}^{2}[1,4,7]$ is

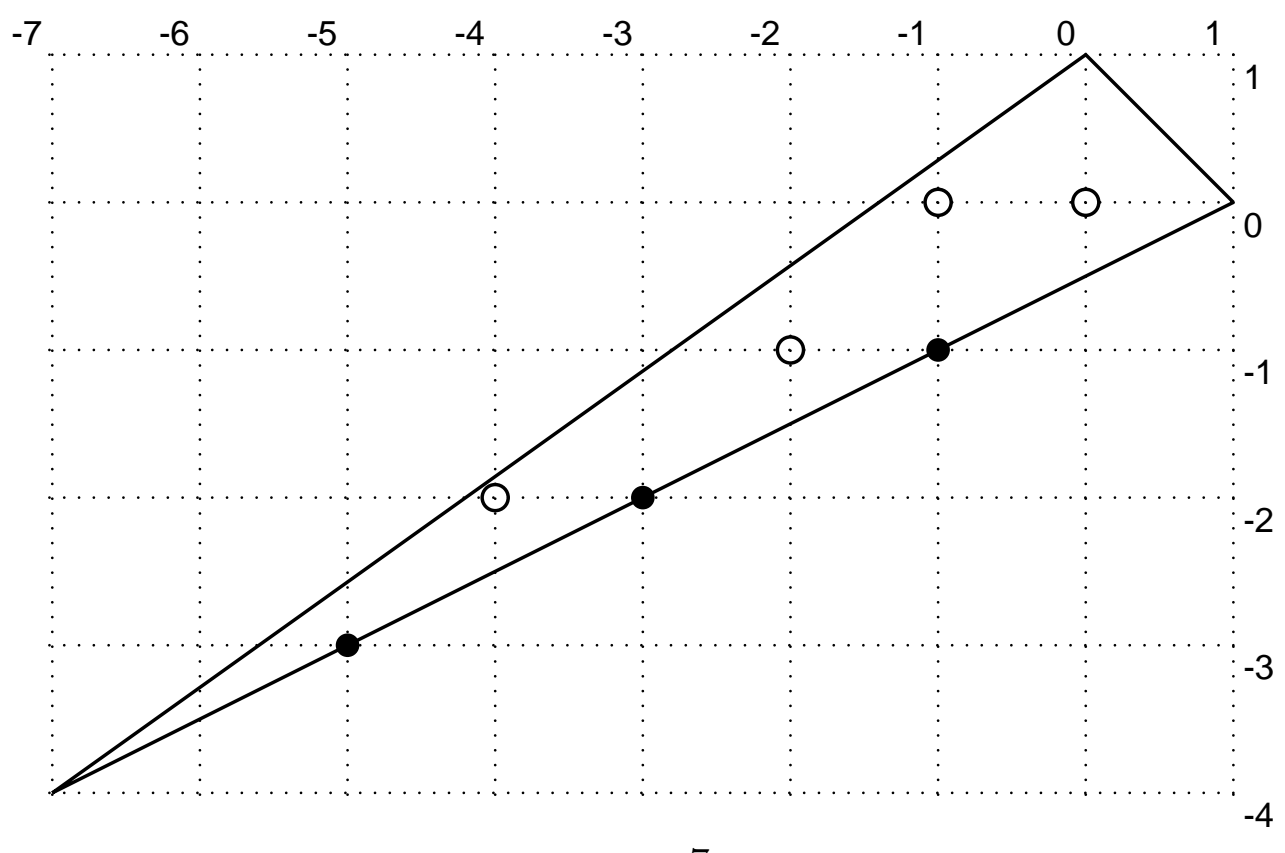


The black circles on this diagram denote integer grid points crossed by the triangle's side; they correspond to the blow-up modes of the singular line $z_{1}=z_{3}=0$ fixed by the $\mathbb{Z}_{4} \subset$ $\mathbb{Z}_{12}$. Physically, such modes are the non-dynamical parameters of the 5D gauge theory such as the inverse gauge coupling $h$ or the masses of hypermultiplets. The open circles denote the grid points completely inside the triangle, which correspond to the homologicallyindependent compact 4-cycles involved in resolving the fixed point itself. Physically, they become the dynamical moduli vector-multiplets of the Coulomb branch of the 5D theory and their number is the rank $r$ of the gauge symmetry.

We are looking for the $S U(M)$ SYM theory which has rank $r=M-1$ and a single nondynamical parameter $h$, hence we need a singularity whose resolution has $M-1$ compact 4-cycles, plus one non-compact cycle responsible for the $h$ parameter. The simplest orbifold of this kind has $a=b=1$ and $N=2 M$,

$$
\mathbb{Z}_{2 M}:\left(z_{1}, z_{2}, z_{3}\right) \mapsto\left(e^{+2 \pi i / 2 M} z_{1}, e^{+2 \pi i / 2 M} z_{2}, e^{-4 \pi i / 2 M} z_{3}\right)
$$

and toric diagram of its blowup is $S L(2, \mathbb{Z})$ equivalent to

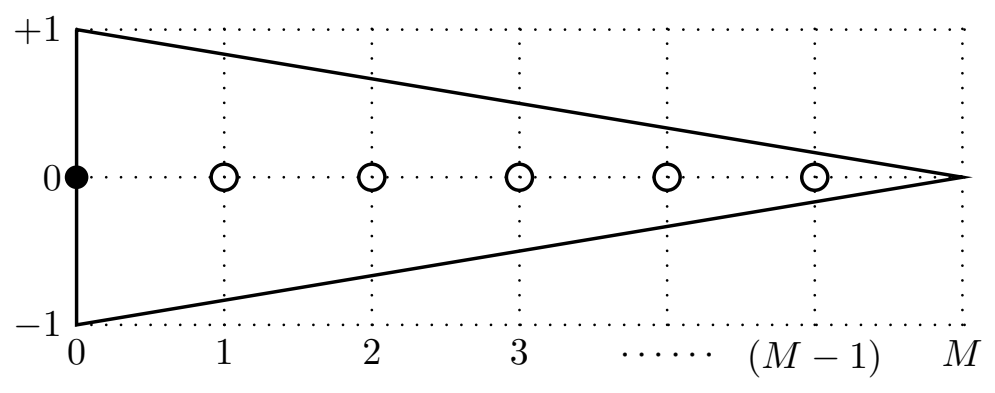

It turns out that this orbifold indeed gives rise to the $S U(M) \mathrm{SYM}$ in 5D. Or rather, it gives rise to this SYM after a partial resolution which blows up the fixed line at $z_{1}=z_{2}=0$ and turns up $h>0($ i. e. $g<\infty)$ but not the $S U(M)$-breaking moduli fields. To see how this works, let us orbifold in two stages

$$
\mathbb{C}^{3} / \mathbb{Z}_{2 M}[1,1,(2 M-2)]=\left(\mathbb{C}^{2} / \mathbb{Z}_{2} \otimes \mathbb{C}\right) / \mathbb{Z}_{M}
$$

and blow up the first stage only. That is, we blowup the $\mathbb{C}^{2} / \mathbb{Z}_{2}$ factor without affecting the 
complex $z_{3}$ plane or resolving the singularities due to the second-stage orbifolding by the $\mathbb{Z}_{M}$ factor. In the linear sigma model description $\left[19\right.$ of this partial blowup, the $\mathbb{C}^{2} / \mathbb{Z}_{2}$ is spanned by 3 complex fields

$$
\begin{aligned}
\left(Z_{1}, Z_{2}, X_{0}\right) \text { modulo } & U(1): Z_{1} \mapsto e^{+i \alpha} Z_{1}, Z_{2} \mapsto e^{+i \alpha} Z_{2}, X_{0} \mapsto e^{-2 i \alpha} X_{0}, \\
\text { constrained to } & \left|Z_{1}\right|^{2}+\left|Z_{2}\right|^{2}-2\left|X_{0}\right|^{2}=D .
\end{aligned}
$$

In string theory, the linear sigma model is in the geometric CY (or rather K3) phase for $D>0$ and in the non-geometric Landau-Ginzburg phase for $D<0$, but only the geometric phase is allowed in the $\mathrm{M}$ theory [12], hence $D \geq 0$ and the parameter space of the blowup is $\mathbb{R}^{+}$rather than $\mathbb{R}$.

The second-stage orbifold action combines the (10) with the $U(1)$ action into

$$
\mathbb{Z}_{M}:\left(X_{0}, Z_{1}, Z_{2}, Z_{3}\right) \mapsto\left(e^{+2 \pi i / M} X_{0}, Z_{1}, Z_{2}, e^{-2 \pi i / M} Z_{3}\right)
$$

which has a fixed line at $X_{0}=Z_{3}=0$ spanned by the $\left(Z_{1}, Z_{2}\right) \in \mathbb{P}^{1}$ of area $D>0$. In other words, the threefold geometry has an $A_{M-1}$ singularity (of complex codimension 2) fibered over the base $B$ which is rational curve with normal bundle $\mathcal{O}(0) \oplus \mathcal{O}(-2)$. In $\mathrm{M}$ theory, this singularity gives rise to the $S U(M)$ SYM theory living in the remaining 7 (real) dimensions comprising $B \times \mathbb{R}_{\text {Minkowski }}^{4,1}$, and after reducing the two compact dimensions of the base $B$, we end up with a 5D $S U(M)$ SYM at finite gauge coupling

$$
g_{5}^{2} \propto \frac{1}{\operatorname{Area}(B)}<\infty, \quad \text { i. e., } \quad h=D>0 .
$$

To study the Coulomb branch of the 5D $S U(M)$ in M theory, we need a complete resolution of the orbifold singularity. In the linear sigma model language, resolving the second-stage $\mathbb{Z}_{M}$ singularity at $X_{0}=Z_{3}=0$ involves $(M-1)$ additional complex fields and a like number of $U(1)$ symmetries and D-term constraints; altogether we have $\left(Z_{1}, Z_{2}, Z_{3} ; X_{0}, X_{1}, \ldots, X_{M-1}\right)$ 
modulo

$$
U(1)^{M}:\left\{\begin{array}{l}
Z_{1} \mapsto \exp \left(i \alpha_{0}\right) Z_{1}, \\
Z_{2} \mapsto \exp \left(i \alpha_{0}\right) Z_{2}, \\
Z_{3} \mapsto \exp \left(i \alpha_{M-1}\right) Z_{3}, \\
X_{0} \mapsto \exp \left(i \alpha_{1}-2 i \alpha_{0}\right) X_{0}, \\
X_{1} \mapsto \exp \left(i \alpha_{2}-2 i \alpha_{1}\right) X_{1}, \\
X_{j} \mapsto \exp \left(i \alpha_{j+1}-2 i \alpha_{j}+i \alpha_{j-1}\right) X_{j} \quad \text { for } j=2, \ldots,(M-2) \\
X_{M-1} \mapsto \exp \left(-2 i \alpha_{M-1}+i \alpha_{M-2}\right) X_{M-1},
\end{array}\right.
$$

and constrained by

$$
\left\{\begin{array}{l}
\left|Z_{1}\right|^{2}+\left|Z_{2}\right|^{2}-2\left|X_{0}\right|^{2}=D>0, \\
\left|Z_{3}\right|^{2}+\left|X_{M-2}\right|^{2}-2\left|X_{M-1}\right|^{2}=D_{M-1}>0, \\
\left|X_{j-1}\right|^{2}+\left|X_{j+1}\right|^{2}-2\left|X_{j}\right|^{2}=D_{j}>0 \quad \text { for } j=1,2, \ldots,(M-2) .
\end{array}\right.
$$

In terms of the toric diagram (11), the $X_{1}, \ldots, X_{M-1}$ are the grid points inside the triangle (open circles), the $X_{0}$ is the grid point on the left side (the black circle) and the $Z_{1}, Z_{2}$ and $Z_{3}$ are the triangle's vertices. 
In real terms, the non-compact CY threefold described by eqs. (16 177) has three independent phases and three independent radii $\left|Z_{1}\right|^{2},\left|Z_{2}\right|^{2}$ and $\left|Z_{3}\right|^{2}$; the constraints (17) impose a concave "floor" keeping these radii away from the origin:

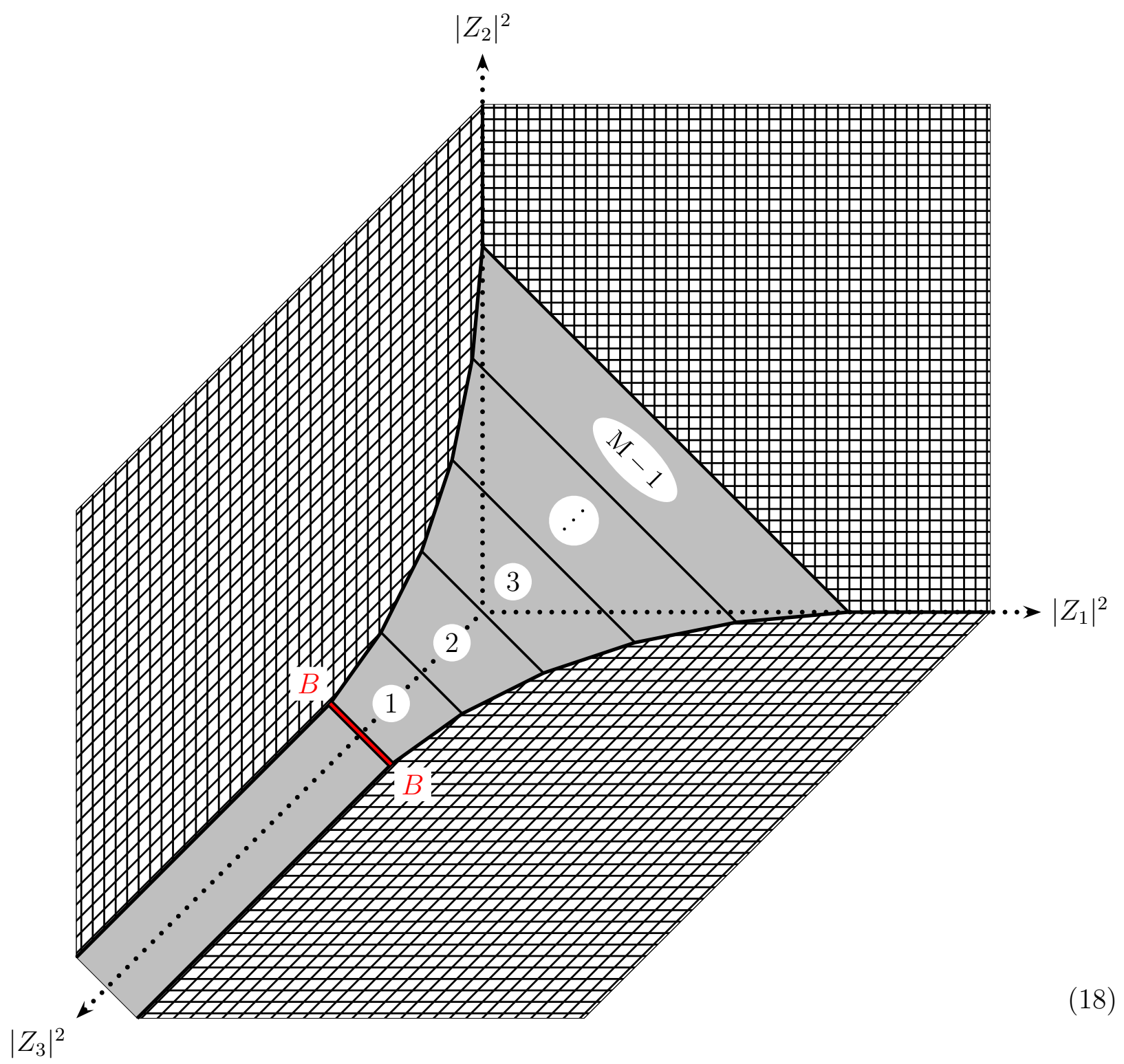

In this picture, the floor "tiles" labeled (1), (2), ., M-1 represent the compact 4cycles $S_{1}, S_{2}, \ldots, S_{M-1}$ which collapse to a common base $B$ when the $S U(M)$ symmetry is restored. From the linear sigma model point of view, it is clear that the base $B$ (the red line 
in $(18)$ ) is a $\mathbb{P}^{1}$ and each 4 -cycle $S_{j}$ is a $\mathbb{P}^{1}$ fibration over $B$. The base area $D$ governs the $5 \mathrm{D}$ gauge coupling while the fiber areas $D_{j}$ control the $S U(M)$ breaking down to the Cartan subgroup $U(1)^{M-1}$; in 5D terms,

$$
D=h, \quad D_{j}=\phi_{j}-\phi_{j+1}
$$

Blowing down the fibers but not the base un-Higgses the $S U(M)$, but what happens when we blow down the base but not the fibers? Mathematically, this is equivalent to making the fibers very large while the base $B$ remains finite, and in that limit, the CY geometry becomes

$$
\mathrm{CY} \longrightarrow \mathbb{C} \times T^{*}\left(\mathbb{P}^{1}\right)
$$

The $T^{*}\left(\mathbb{P}^{1}\right)$ does not admit flop transitions to anything else, which means that the resolved orbifold also does not have any flop transitions to a physically different phase with $h<0$. Thus, we conclude that the UV completion of the 5D $S U(M)$ SYM theory via M theory on the $\mathbb{C}^{3} / \mathbb{Z}_{2 M}$ orbifold has parameter space $\mathbb{R}^{+}$and no phase transitions.

In the following sections, we shall obtain the same parameter space for the UV completions via 5-brane webs in the type IIB string theory or via dimensional deconstruction, provided the SYM theory has Chern-Simons level $k_{\mathrm{cs}}=M$. To compare the results, we need the Chern-Simons level of the SYM derived from the M theory on the $\mathbb{C}^{3} / \mathbb{Z}_{2 M}$. We shall see momentarily that this Chern-Simons level is indeed $k_{\mathrm{cs}}=M$, hence the $\mathbb{R}^{+}$parameter space is appears to be the intrinsic property of the $S U(M)$ SYM theory at this Chern-Simons level regardless of the UV completion.

As a warm-up exercise, consider the $S U(2)$ case. In 5D, the $S U(2)$ SYM does not have Chern-Simons interactions (due to lack of a cubic invariant), but thanks to $\pi_{4}(S U(2))=\mathbb{Z}_{2}$ there is a discrete vacuum angle $(\theta=0$ or $\theta=\pi)$ and hence two distinct quantum theories. Their respective $g \rightarrow \infty$ superconformal limits are called the $E_{1}$ and the $\tilde{E}_{1}$; they belong to the $E$ series of the rank 1 SCFTs whose global symmetries form the exceptional $E_{n}$ groups, hence the name [6, []. In M theory, an $E_{n}$ SCFT shows up when a del Pezzo surface $\mathcal{B}_{n}$

\footnotetext{
${ }^{1}$ The resolved orbifold does have flop transitions to negative fiber areas $D_{j}<0$, but these are just the Weyl symmetries of the $S U(M)$.
} 
collapses to a point [6, 7]; the surfaces $\mathcal{B}_{1}$ and $\widetilde{\mathcal{B}}_{1}$ giving rise to the $E_{1}$ and the $\tilde{E}_{1}$ happen to be $\mathbb{P}^{1}$ fibrations over the $\mathbb{P}^{1}$ base without bad fibers. In other words, they are Hirzebruch ruled surfaces

$$
\mathbb{F}_{n} \stackrel{\text { def }}{=} \mathbb{P}(\mathcal{O}(0) \oplus \mathcal{O}(n))
$$

specifically, $\mathcal{B}_{1}=\mathbb{F}_{0}=\mathbb{P}^{1} \times \mathbb{P}^{1}$ and $\widetilde{\mathcal{B}}_{1}=\mathbb{F}_{1}$.

When a Hirzebruch surface $\mathbb{F}_{n}$ collapses to a line rather than a point because its base $B$ retains finite area while the fiber area shrinks to zero, the $\mathrm{M}$ theory on the resulting singularity produces an $S U(2)$ SYM with a finite gauge coupling instead of an SCFT. This works for any fibration degree $n$, but the parity of $n$ affects the vacuum angle $\mathrm{f}$ :

$$
\theta=n \pi \text { modulo } 2 \pi \text {. }
$$

In the $\mathbb{C}^{3} / \mathbb{Z}_{2 M}$ orbifold model for $M=2$, the compact 4-cycle of the fully resolved singularity is the blown-up weighted projective space $\mathbb{W} \mathbb{P}^{2}[1,1,2]$. The blow-up removes the $\mathbb{Z}_{2}$ orbifold singularity of the $\mathbb{W P}^{2}[1,1,2]$ itself and turns it into the Hirzebruch surface $\mathbb{F}_{2}$ of degree 2 . For the partially resolved $\mathbb{Z}_{4}$ singularity, the $\mathbb{F}_{2}$ collapses to its base $B$ and the $S U(2)$ is un-Higgsed. According to eq. (22), the vacuum angle of this $S U(2)$ is zero rather than $\pi$.

The orbifold models with $M>2$ have several compact 4 -cycles $S_{1}, \ldots, S_{M-1}$, cf. the picture (18). Let us calculate their triple intersections $S_{j} \cdot S_{k} \cdot S_{\ell}$ in the fully resolved CY3fold. According to [8], this will give us the prepotential for the $M-1$ abelian vector multiplets of the Coulomb branch of the 5D SYM: In terms of the Cartan moduli $a_{i}=\phi_{i+1}-\phi_{i}$,

$$
\mathcal{F}_{3} \equiv \text { the cubic part of } \mathcal{F}=\frac{1}{6}\left[\sum_{i, j=1}^{M-1} C^{i j} a_{i} S_{j}\right]^{3}
$$

where $C^{i j}$ is the inverse Cartan matrix of the $S U(M)$, or in terms of the eigenvalues $\phi_{i}$,

$$
\mathcal{F}_{3}=\frac{1}{6}\left[\sum_{j=1}^{M-1}\left(\phi_{j+1}+\cdots+\phi_{M}\right) S_{j}\right]^{3}
$$

Physically, the result of this calculation should agree with the $S U(M)$ Coulomb branch

\footnotetext{
${ }^{2}$ As we will see in the next section from the type IIB web perspective the Hirzebruch surface $\mathbb{F}_{n}$ for $n>2$ must be accompanied by other 4-cycle inside a CY3-fold.
} 
prepotential (4), and we shall see momentarily that this is indeed the case for $k_{\mathrm{cs}}=M$.

The toric diagram of (11) of the orbifold implies that the 4 -cycle $S_{j}$ is a Hirzebruch surface $\mathbb{F}_{2 j}$. Indeed, in the linear sigma model language ( $c f$. eqs. (16 [17) and the picture (18)), $S_{j}$ is the locus of $X_{j}=0$ where sigma model reduces to just 4 fields $\left(Z_{1}, Z_{2}, X_{j-1}, X_{j+1}\right)$ with 2 abelian symmetries and 2 constraints:

$$
\begin{aligned}
U(1)^{2}:\left(Z_{1}, Z_{2}, X_{j-1}, X_{j+1}\right) & \mapsto\left(e^{i \alpha_{0}} Z_{1}, e^{i \alpha_{0}} Z_{2}, e^{i\left(\alpha_{j}-2 j \alpha_{0}\right)} X_{j-1}, e^{i \alpha_{j}} X_{j+1}\right), \\
\left|Z_{1}\right|^{2}+\left|Z_{2}\right|^{2}-2 j\left|X_{j-1}\right|^{2} & =D+2 D_{1}+4 D_{2}+\cdots+(2 j-2) D_{j-1}>0, \\
\left|X_{j-1}\right|^{2}+\left|X_{j+1}\right|^{2} & =D_{j}>0,
\end{aligned}
$$

which clearly describes the ruled surface $\mathbb{F}_{2 j}$. The second homology of this surface is spanned by the fibration base $B_{j}$ and the fiber $F_{j}$ which intersect according to

$$
B_{j} \cdot B_{j}=-2 j, \quad B_{j} \cdot F_{j}=1, \quad F_{j} \cdot F_{j}=0
$$

and the canonical class dual to the first Chern class $c_{1}\left(S_{j}=\mathbb{F}_{2 j}\right)$ is

$$
-K_{S_{j}}=2 B_{j}+(2 j+2) F_{j}
$$

In the Calabi-Yau context, this canonical class controls the intersections of $S_{j}$ with lines equivalent to rational curves within the $S_{j}$ itself:

$$
\forall C \subset S_{j}:\left[S_{j} \cdot C\right]_{\mathrm{CY}}=\left[K_{S_{j}} \cdot C\right]_{S_{j}}
$$

The curves of interest for our purposes are the intersections of $S_{j}$ with itself or with the other $S_{i}$. The self-intersection $S_{j} \cdot S_{j}$ can be found by deforming the $S_{j}$ inside the CalabiYau threefold and finding the vanishing locus of the deformation. The deformations normal to the $S_{j}$ are sections of the canonical class $K_{S_{j}}$ and vanish on the canonical class itself. Hence, $S_{j} \cdot S_{j}=K_{S_{j}}$ and therefore (thanks to eq. (28))

$$
S_{j} \cdot S_{j} \cdot S_{j}=K_{S_{j}} \cdot K_{S_{j}}=8 .
$$

Besides itself, the cycle $S_{j}$ intersects its nearest neighbors $S_{j \pm 1}$ but not other $S_{i}$ with $i \neq$ 
$j, j \pm 1$ - this is obvious from the diagram (18). For the neighbors,

$$
S_{j} \cdot S_{j+1}=B_{j+1}=B_{j}+2 j F_{j}
$$

and therefore

$$
\begin{aligned}
S_{j} \cdot S_{j+1} \cdot S_{j+1} & =B_{j+1} \cdot K_{S_{j+1}}=+2 j, \\
S_{j} \cdot S_{j+1} \cdot S_{j} & =\left(B_{j}+2 j F_{j}\right) \cdot K_{S_{j}}=-(2 j+2) .
\end{aligned}
$$

All other triple intersections of the $S_{j}$ cycles vanish, hence substituting eqs. (29) and (31 32) into eq. (24) we arrive (after some messy algebra) at the cubic prepotential

$$
\mathcal{F}_{3}=\frac{1}{12} \sum_{i j}\left|\phi_{i}-\phi_{j}\right|^{3}+\frac{M}{6} \sum_{i} \phi_{i}^{3} .
$$

As promised, this prepotential is exactly as in eq.(四) for $k_{\mathrm{cs}}=M$. Thus, we identify the low-energy limit of the $\mathrm{M}$ theory on the resolved orbifold as the Coulomb branch of the $S U(M)$ SYM at the Chern-Simons level $k_{\mathrm{cs}}=M$.

The SYM theories with other Chern-Simons levels also have UV completions via M theory compactified on singular CY threefolds, but the singularities for $\left|k_{\mathrm{cs}}\right| \neq M$ are not orbifolds. They also have $\mathbb{R}$ rather than $\mathbb{R}^{+}$parameter spaces, and they do have flop transitions to physically different phases for $h<0$ for $M-k$ odd. In the next section, we shall derive these results from the dual description in terms of $(p, q) 5$-brane webs of the IIB superstring. 


\subsection{Five-Brane Webs in IIB Superstring Theory}

The type IIB superstring theory has BPS five-branes with any mutually-prime combinations $(p, q)$ of the R-R and NS-NS magnetic charges. A brane web [9, 11] comprises a network of such five-branes spanning $1+4$ common infinite dimensions $\left(x^{0}, x^{1}, x^{2}, x^{3}, x^{4}\right)$ while the fifth space dimension of each $(p, q)$ brane is a line in the common $\left(x^{5}, x^{6}\right)$ plane $\left(x^{7}=x^{8}=x^{9}=0\right)$ in the direction

$$
x^{5}+i x^{6}=p+q \tau
$$

where $\tau_{\mathrm{IIB}}=\frac{4 \pi i}{g^{2}}+\frac{a}{2 \pi}$ is the "holomorphic" type IIB string coupling. The lines may be finite or infinite in one direction; generally, they split and join each other at trivalent vertices where eqs.

$$
\sum_{i=1}^{3} p_{i}=\sum_{i=1}^{3} q_{i}=0
$$

are required by the charge conservation. Together, eqs. (34) and (35) assure the mechanical stability of the five-brane web - as well as the $\mathcal{N}=1$ supersymmetry of the effective $5 \mathrm{D}$ theory which lives on it.

The 5D theory living on a generic brane web is an abelian gauge theory coupled to massive charged particles arising from the open strings (or webs of open strings) ending on the five-branes. The rank of this 5D theory is the number of loops in the graph of the brane web in the 56 plane, and the moduli correspond to normal movements of the finite-length internal lines in this graph while the semi-infinite external lines remain fixed. Moving the external lines of the graph relative to each other corresponds to changing the non-dynamic parameters of the 5D theory such as $h$ or quark masses [9].

A movement which puts several parallel lines directly on top of each other un-Higgses a non-abelian gauge symmetry as the zero-length strings ending on coincident 5-branes give rise to massless charged vectors $W_{i j}$. The $5 \mathrm{D}$ gauge coupling $g_{5}^{2}$ is inversely proportional to the length of this stack of coincident branes and becomes infinitely strong for an infinitesimally short stack [9]. More generally, a loop (or several loops) of branes collapsing to a point in the 56 plane rather than a finite line gives rise to a superconformal field theory in $5 \mathrm{D}$, similar to a 4-cycle (or several 4-cycles) in M theory collapsing to a single point. 
Actually, the five-brane webs in type IIB superstring theory are dual to non-compact toric CY3-folds in M theory [10]. To see this duality, note that a Calabi-Yau threefold is generally a $T^{3}$ fibration over $S^{3}$ or some other real three-fold [24]. In the non-compact case of a resolved singularity such as $\mathbb{C}^{3} / \mathbb{Z}_{N}$, the fibrations base is topologically $\mathbb{R}^{3}$ or $\mathbb{R}^{2} \times \mathbb{R}^{+}$ while the fiber is generically $T^{2} \times \mathbb{R}$ but the $T^{2}$ degenerates to a $1 \mathrm{D}$ circle along a web of real lines within a single $\mathbb{R}^{2}$ plane inside the $\mathbb{R}^{3}$ base. The duality acts fiber-wise and turns $\mathrm{M}$ theory on a $T^{2}$ into type IIB superstring on a circle. A degenerate $T^{2}$ turns into a IIB fivebrane along the locus of degeneration — the real line in the base, times the $\mathbb{R}^{4,1}$ Minkowski space - whose $(p, q)$ charges depend on the particular 1-cycle $p \alpha_{1}+q \alpha_{2}$ of the $T^{2}$ which shrinks to zero length [10]. Thanks to the holomorphy of the Calabi-Yau, the direction of the degeneration line within the $\mathbb{R}^{2}$ plane in the base follows the collapsing 1-cycle, hence the dual $(p, q)$ five-brane satisfies the charge-direction equation (34) [10] .

For example, in the $\mathbb{C}^{3} / \mathbb{Z}_{2 M}$ model of the previous section, the fibration base is shown in the diagram (18): The $T^{2}$ degenerates along the solid lines of the diagrams, and the whole network of these lines is restricted to a single gray surface (the "floor"), which topologically is an $\mathbb{R}^{2}$ plane spanned by the $\left|Z_{1}\right|^{2} \pm\left|Z_{2}\right|^{2}$. After the $\mathrm{M} \leftrightarrow \mathrm{IIB}$ duality, the five-brane web looks just like the floor diagram of (18), namely

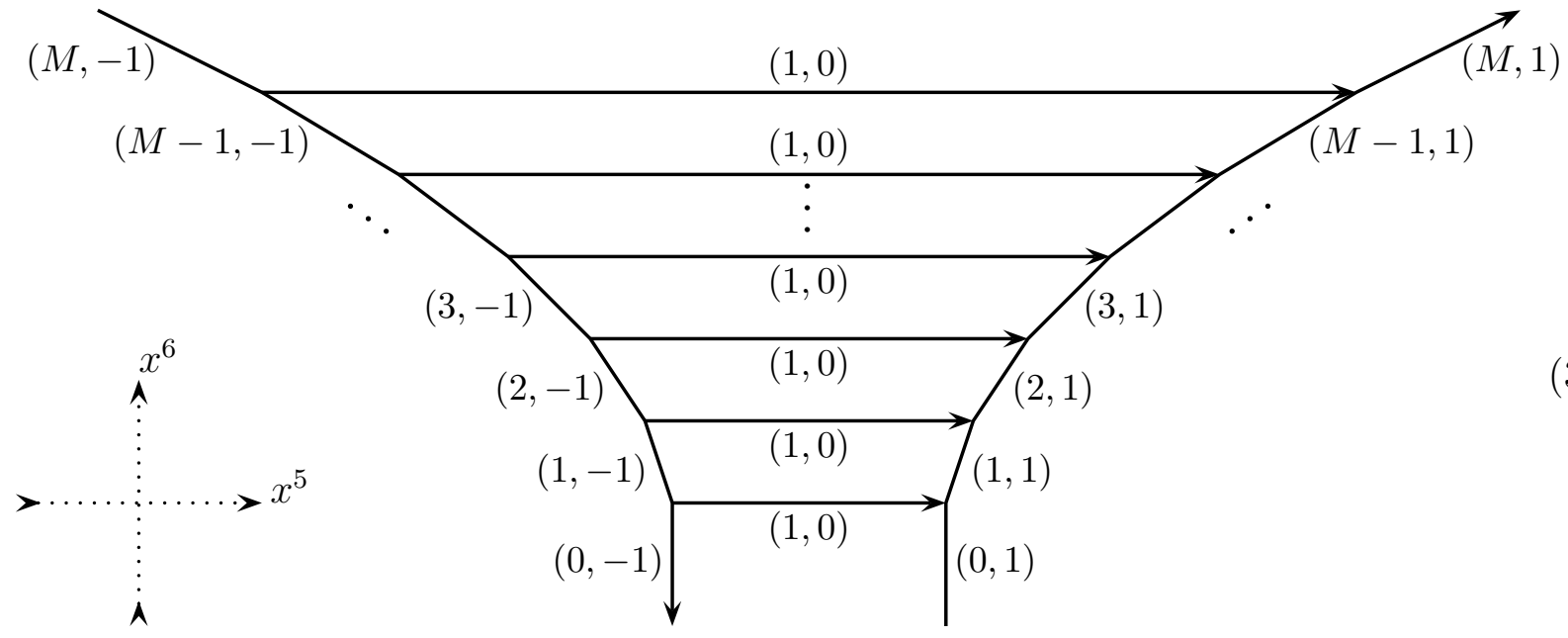

where the $(p, q)$ charges — and hence the precise directions - of the diagonal lines follow from eqs. (35). 
The graphic dual[ of the web (36) looks like

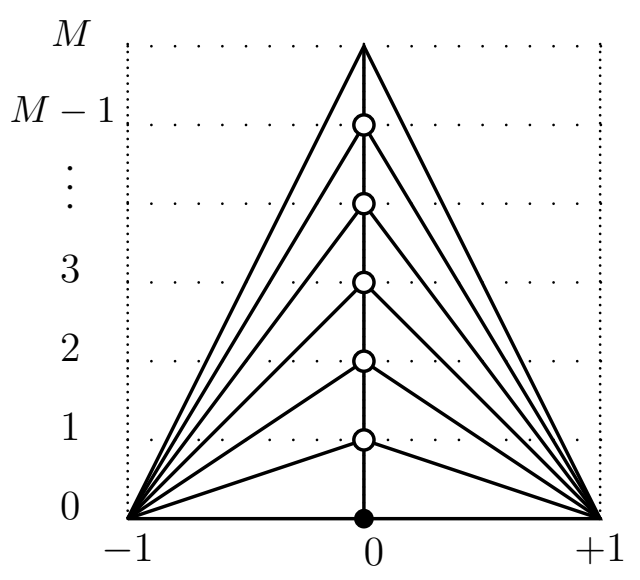

which is clearly the triangulated toric diagram (11) of the $\mathbb{C}^{3} / \mathbb{Z}_{2 M}$ orbifold. This is an example of a general rule: If type IIB string theory on a five-brane web $W$ is dual to M theory on a resolved Calabi-Yau singularity $M$, then the graphic dual of the web $W$ is the triangulated toric diagram of $M$. Indeed, the loops of the web $W$ are dual to the compact 4-cycles of $M$, which are represented on the toric diagram by internal vertices dual to those loops. Likewise, the lines separating the loops of $W$ are dual to intersections of $M$ 's 4-cycles, and the graphically dual lines on the toric diagram connect the corresponding vertices [9, 10, 11].

\footnotetext{
${ }^{3}$ The graphic duality maps a planar graph $A$ onto another planar graph $B$ such that the loops (faces) of $A$ map onto the vertices of $B$ and vice verse, and the corresponding lines of the two graphs are perpendicular to each other. By abuse of notations, a graphic dual of a five-brane web is the graphic dual of its 56 plane.
} 
For example, consider a rank $1 \mathrm{CY}$ singularity of M theory where the collapsing 4-cycle is a Hirzebruch surface $\mathbb{F}_{n}$. The toric diagrams of the $\mathbb{F}_{n}$ are

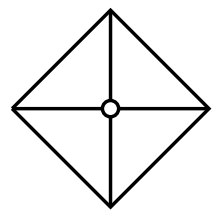

$\mathbb{F}_{0}$

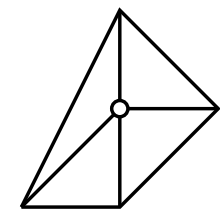

$\mathbb{F}_{1}$

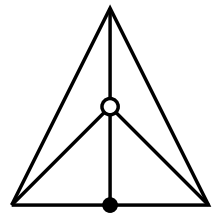

$\mathbb{F}_{2}$

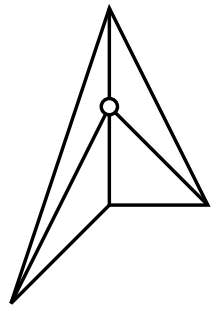

$\mathbb{F}_{3}$

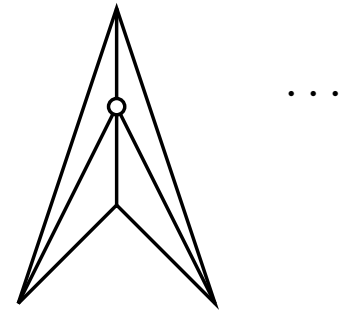

$\mathbb{F}_{4} \quad \cdots$

and the IIB five-brane webs dual to the resolved CY3-fold singularities form graphs dual to the (38), namely

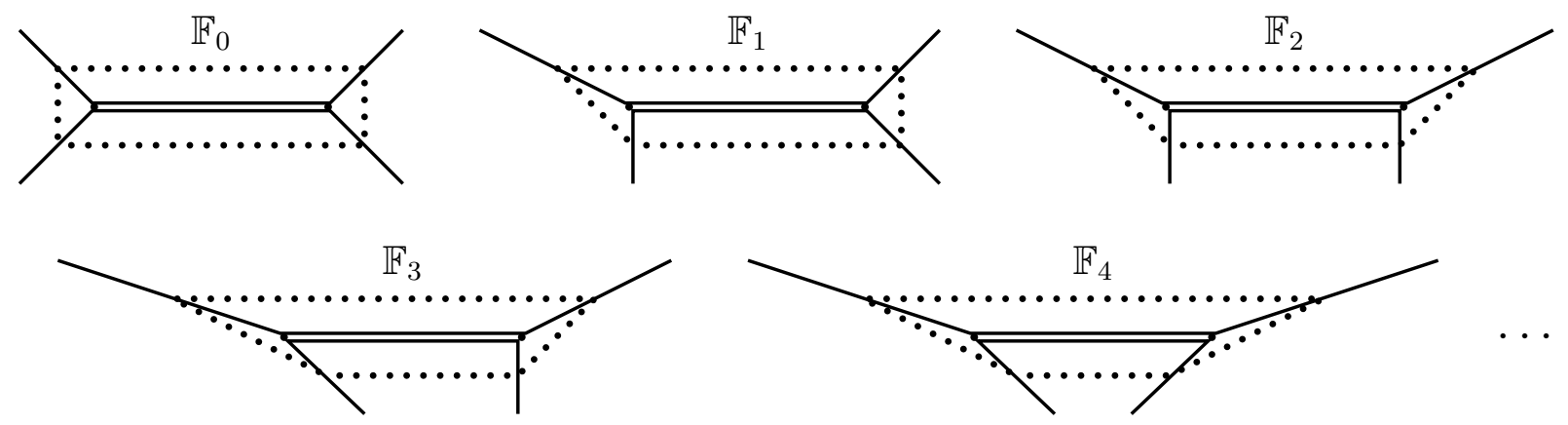

where the dotted lines describe the Coulomb branch of the 5D theory and the solid lines the un-Higgsed $S U(2)$ limit. Note that for $n>2$ the toric diagram is not convex and the web graph has two converging external lines. Clearly, these lines cannot extend to infinity, which means the $\mathbb{F}_{n>2}$ web can only be a subset of a bigger web of rank $r>1$, or in the $\mathrm{M}$ theory language, a $\mathrm{CY}$ singularity with an $\mathbb{F}_{n>2}$ cycle must have other compact cycles as well.

For each web diagram (39), the length of the doubled-up dashed line in the middle of the graph gives the inverse gauge coupling $h$ of the un-broken 5D $S U(2)$. We change this coupling by moving the external legs relative to each other, and for the stand-alone $\mathbb{F}_{0}, \mathbb{F}_{1}$ 
and $\mathbb{F}_{2}$ webs the complete parameter space includes the following configurations:

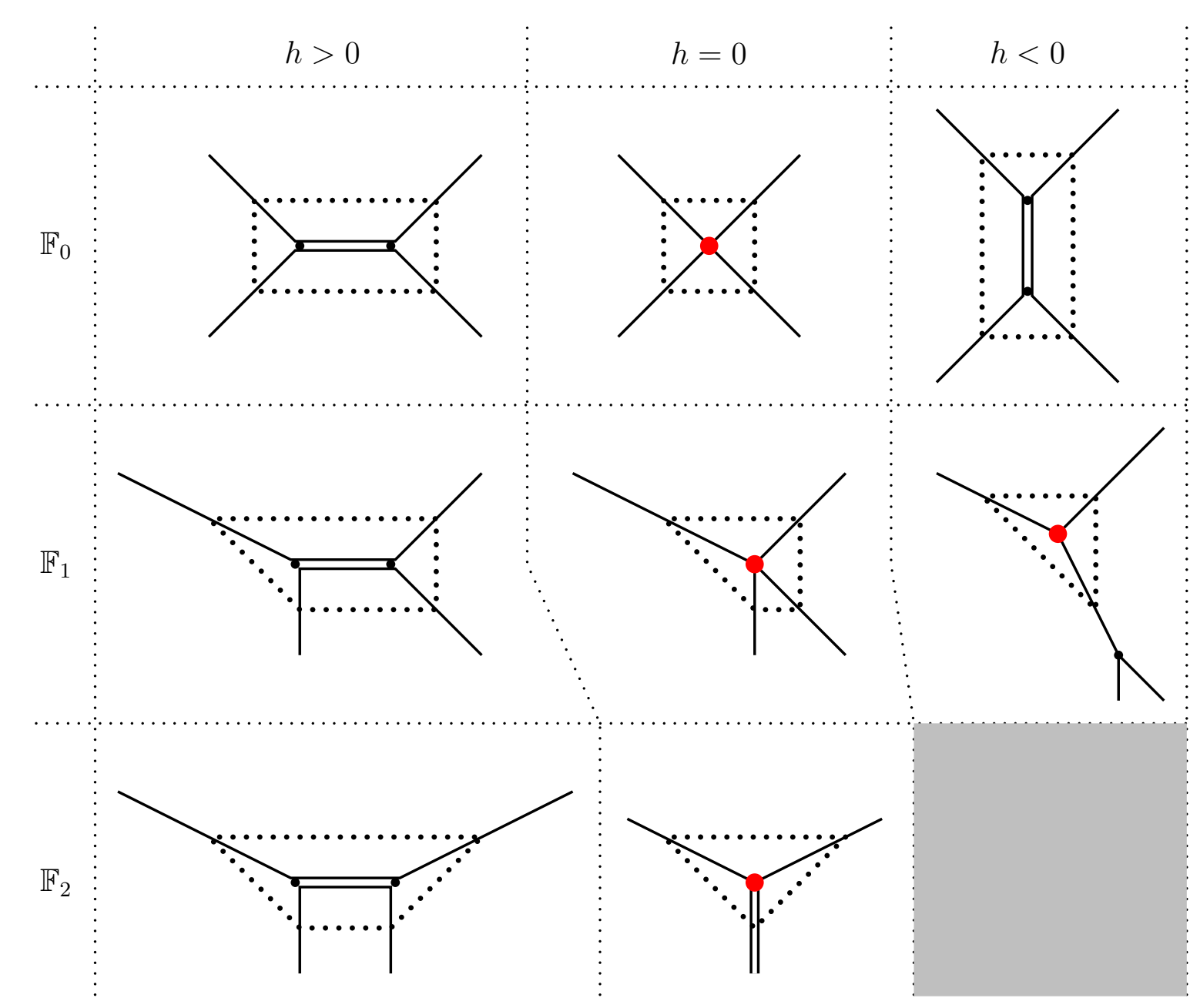

$(40)$

where the red circles $\bullet$ denote loops collapsing to points and giving rise to superconformal theories in 5D. We see that all three webs have a superconformal point at $h=0$, but the flop transition to $h<0$ is very different: For the $\mathbb{F}_{0}$, the flop is a symmetry and the same $S U(2)$ SYM with $\theta=0$ obtains for both $h>0$ and $h<0$. The $\mathbb{F}_{1}$ has two different phases: The $S U(2)$ SYM with $\theta=\pi$ for $h>0$ and the $E_{0}$ superconformal theory (the red circle) plus some massive particles for $h<0$. Finally, the $\mathbb{F}_{2}$ web does not flop at all: $h$ is the distance between two parallel external legs, and it cannot go negative no matter what. This confirms the M-theory result: The parameter space of the $\mathbb{F}_{2}$ singularity is $\mathbb{R}^{+}$rather than $\mathbb{R}$ and there are no flop transitions. 
Now consider the $S U(M)$ theories with $M>2$. The five-brane web (36) dual to the $\mathbb{C}^{3} / \mathbb{Z}_{2 M}$ orbifold of $\mathrm{M}$ theory behaves exactly line the $\mathbb{F}_{2}$ web for $M=2$ : A quick look at the web of the un-Higgsed $S U(M)$

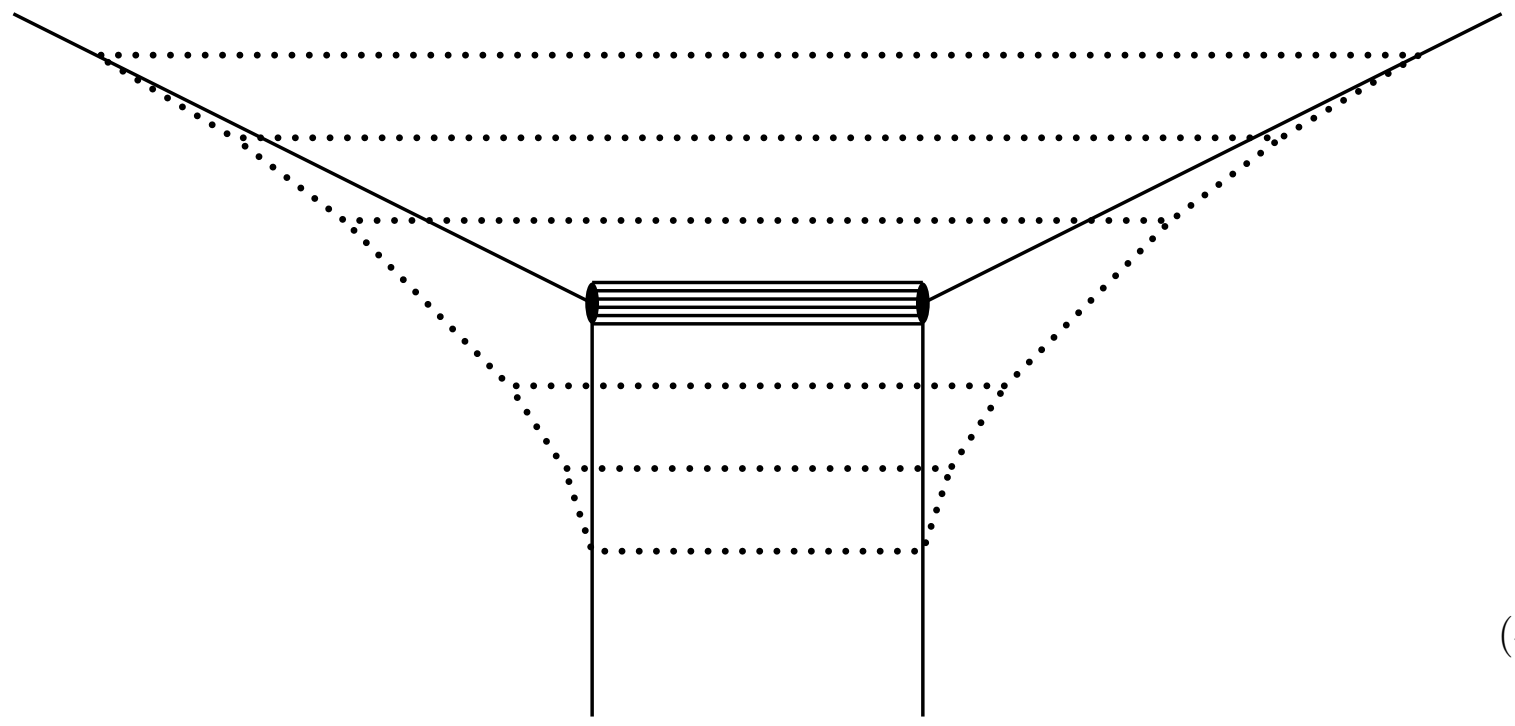

shows that the inverse gauge coupling $h$ is the distance between two parallel external legs, hence $h \geq 0$ no matter what and $\mathbb{R}^{+}$parameter space. But just as in the $M=2$ case, there are other webs which allow flop transitions between an $S U(M)$ SYM for $h>0$ and some other 5D theory for $h<0$. Generally, for $h>0$ all such webs are $S L(2, \mathbb{Z})$ equivalent to

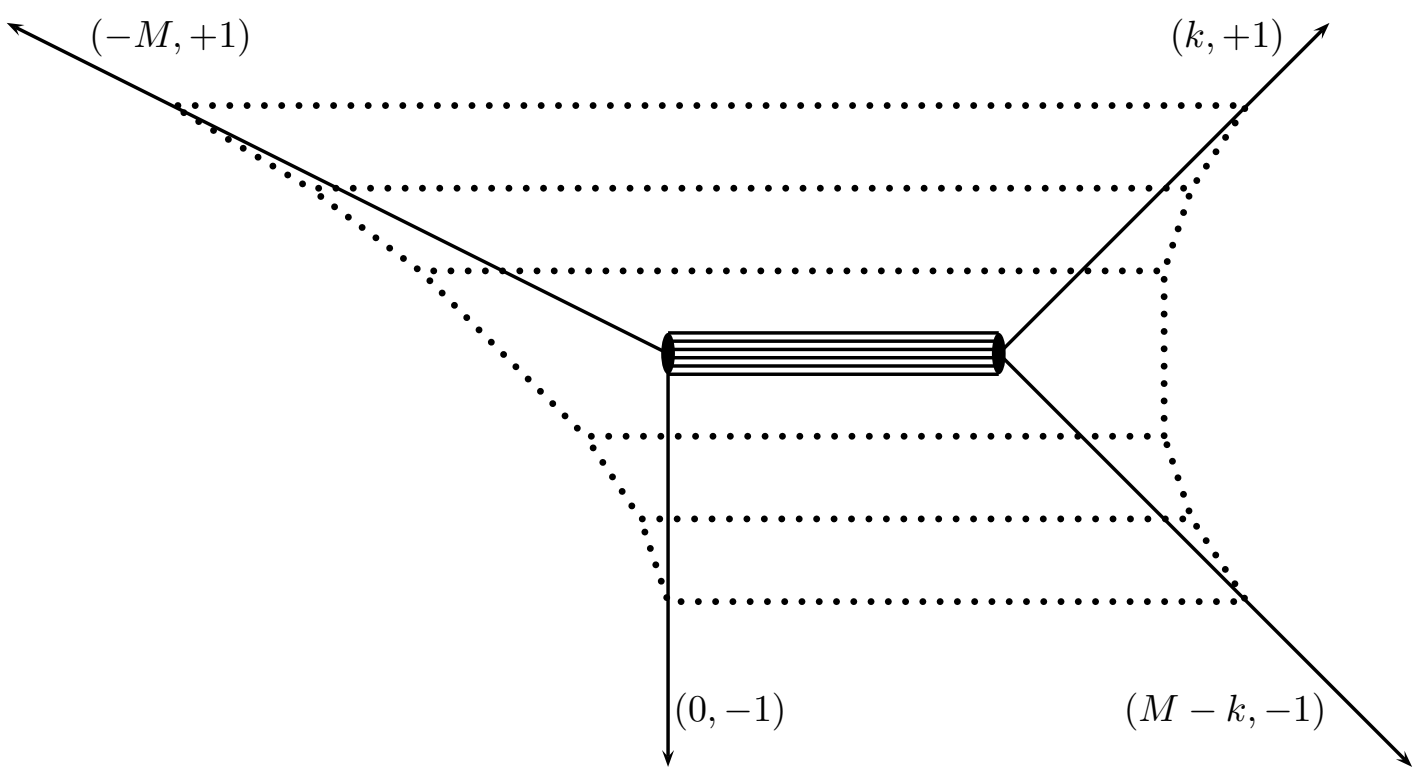


for some integer $k$ between $-M$ and $+M$, and two parallel external legs - and hence no flop transitions to $h<0$ - only for $k= \pm M$.

Physically, $k$ is the Chern-Simons level of the 5D theory. Indeed, consider the Coulomb branch of the web depicted by the dotted lines on fig. (42). Let $y_{1}<y_{2}<\cdots y_{M}$ denote the $x^{6}$ positions of the $(1,0)$ branes (the vertical positions of the horizontal lines) and $t_{1}, t_{2}, \ldots, t_{M}$ their lengths in the $x^{5}$ direction; thanks to eqs. (35),

$$
t_{i}=h+k \phi_{i}+\sum_{j=1}^{M}\left|y_{i}-y_{j}\right| .
$$

From the 5D point of view, the $y_{i}$ are the eigenvalues $\phi_{i}$ of the adjoint scalar field breaking the $S U(M) \rightarrow U(1)^{M-1}$ while the $t_{i}$ are the inverse gauge couplings of the surviving abelian symmetries. Thus, we interpret the web geometry relations (43) in terms of the 5D gauge coupling formulae (5), which immediately identifies $k$ as the Chern-Simons level $k_{\mathrm{cs}}$.

For $k \neq \pm M$, the external legs of the web diagram (42) are diverging rather than parallel and there is a flop transition to $h<0$. As an example, consider the case of $S U(3)$ at $k_{\mathrm{cs}}=2$ where the web flops to an exotic rank 2 SCFT (plus massive junk):
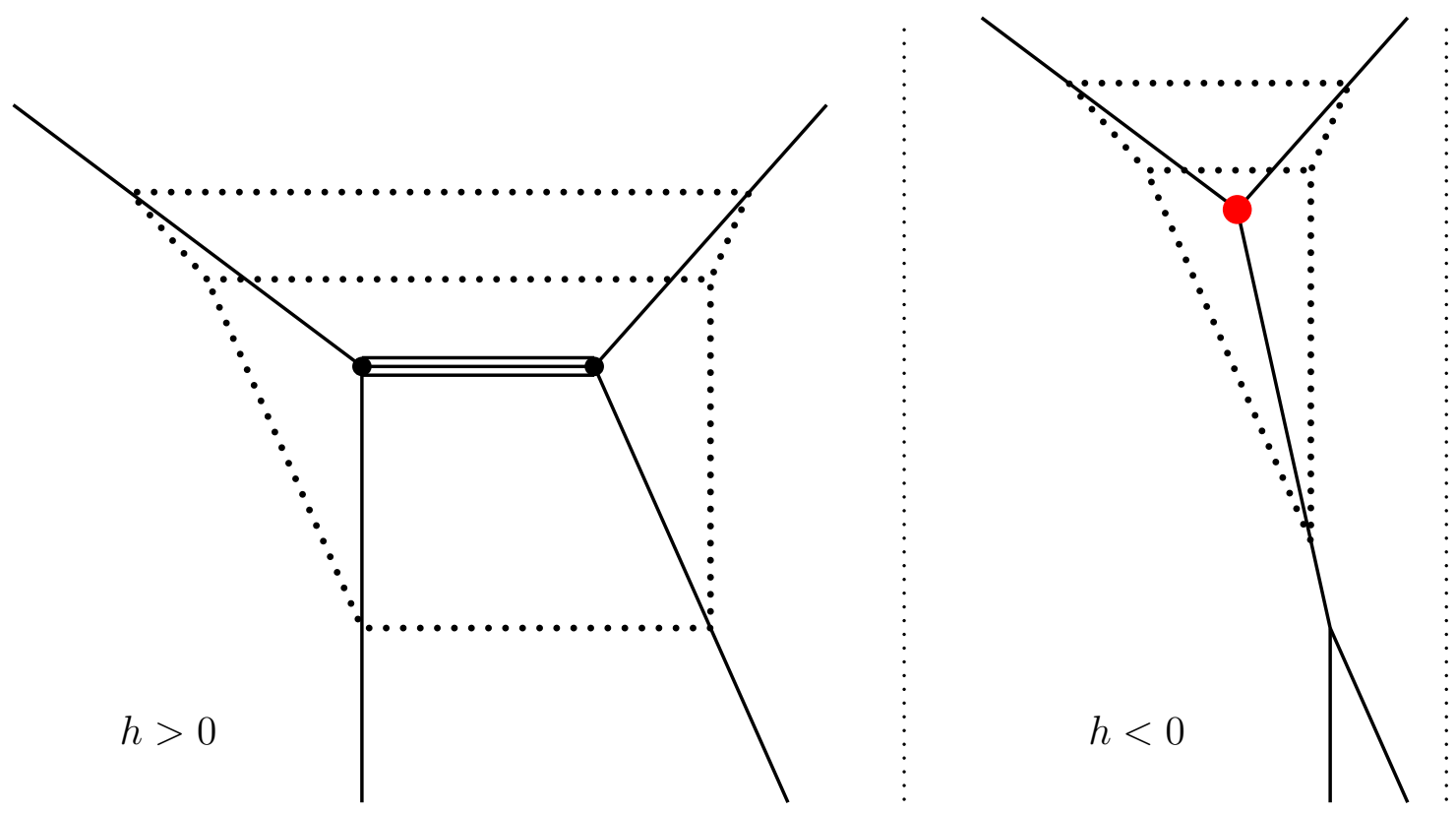

In the dual $\mathrm{M}$ theory, this flop changes the triangulation of the toric diagram of the $\mathrm{CY}$ 
singularity:

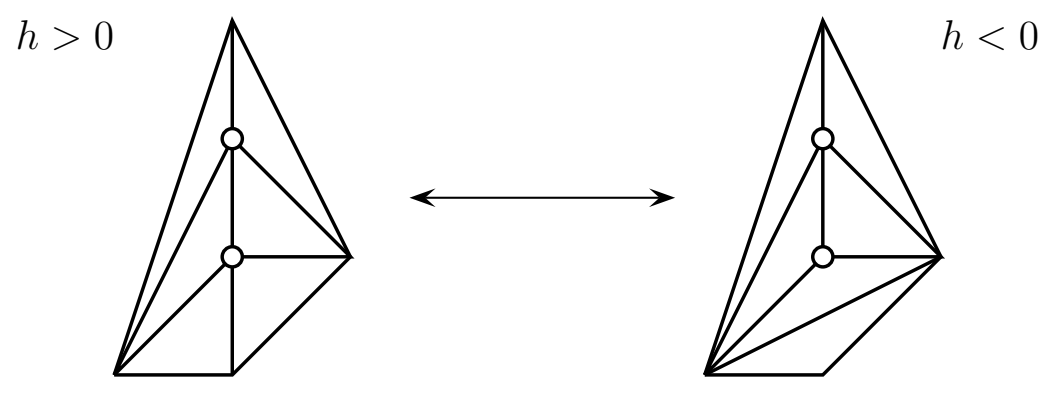

Focusing on the 5D SCFT for $h<0$ and ignoring the massive stuff, we truncate the toric diagram and the web to
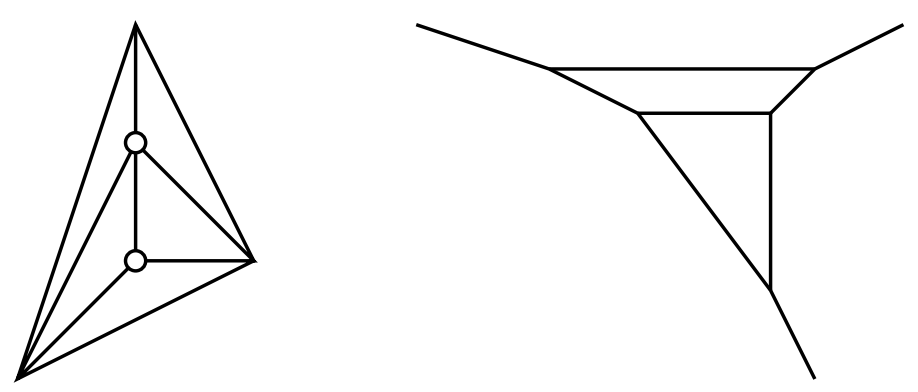

In $\mathrm{M}$ theory, this describes 2 compact 4 -cycles - an $\mathbb{F}_{3}$ and a $\mathbb{P}^{2}$ - intersecting each other along a $\mathbb{P}^{1}$. The triangular shape of the toric diagram indicates that the singularity containing these cycles is an orbifold fixed point; an $S L(2, \mathbb{Z})$ transform maps the triangle's vertices to respectively $(0,1),(1,0)$ and $(-1,-3)$, which pinpoint a particular orbifold, namely the $\mathbb{C}^{3} / \mathbb{Z}_{5}[1,1,3]$. On the IIB side, the truncated web has only three external lines and thus no non-dynamical parameters (the $h<0$ parameter of the full web affects the massive junk but decouples from the SCFT in question). Hence, when both loops of the web graph shrink in area, they collapse to a single point rather than a stack of coincident lines. In $\mathrm{M}$ theory terms, this means the singularity cannot be partially resolved into a line; it has to be resolved in full or not at all. And in 5D terms, this means an exotic SCFT which does not follow from the $g_{5}=\infty$ limit of an ordinary gauge theory.

Note that the $\mathbb{C}^{3} / \mathbb{Z}_{5}$ orbifold in M theory describes the SCFT degrees of freedom of the $h<0$ phase of the $S U(3)$ (at $k_{\mathrm{cs}}=2$ ) but without additional particles with $h$-dependent masses one cannot flop back into the $h>0$ SYM phase. The full 5D theory follows from 
M theory on a more complicated singularity whose toric diagram is (45) rather than (46), and this singularity is not of the orbifold type. Indeed, for all of the $S U(M)$ theories at Chern-Simons levels $k \neq \pm M$, embedding into M theory requires a more complicated CY singularity than just a $\mathbb{C}^{3} / \mathbb{Z}_{N}$ orbifold because the the toric diagrams dual to their (42) webs are proper quadrangles rather than triangles. The $k_{\mathrm{cs}}= \pm M$ theories are fortunate exceptions from this rule.

Conversely, M theory on orbifold singularities with eigenvalues other than $(1,1,2 M-2)$ produce 5D theories more complicated than a non-abelian SYM. For example, for odd $N=$ $2 M+1$, the $\mathbb{C}^{3} / \mathbb{Z}_{N}$ orbifold with eigenvalues $(1,1,2 M-1)$ is an exotic SCFT of rank $M$ : The good old $E_{0}$ for the $\mathbb{Z}_{3}$ orbifold, the rank 2 theory depicted on fig. (46) for the $\mathbb{Z}_{5}$, or a higher-rank generalization of the same pattern for odd $N>5$ : The compact 4-cycles are $\left\{\mathbb{F}_{N-2}, \mathbb{F}_{N-4}, \cdots, \mathbb{F}_{3}, \mathbb{P}^{2}\right\}$, and when all the cycles shrink, they collapse to a single point rather than a line. Another example is the $\mathbb{Z}_{12}$ orbifold with eigenvalues $(1,4,7)$ whose toric diagram is shown in fig. (9); its type IIB brane web dual follows via graphic duality: Modulo a choice of triangulations and an $S L(2, \mathbb{Z})$ rotation, the web looks like

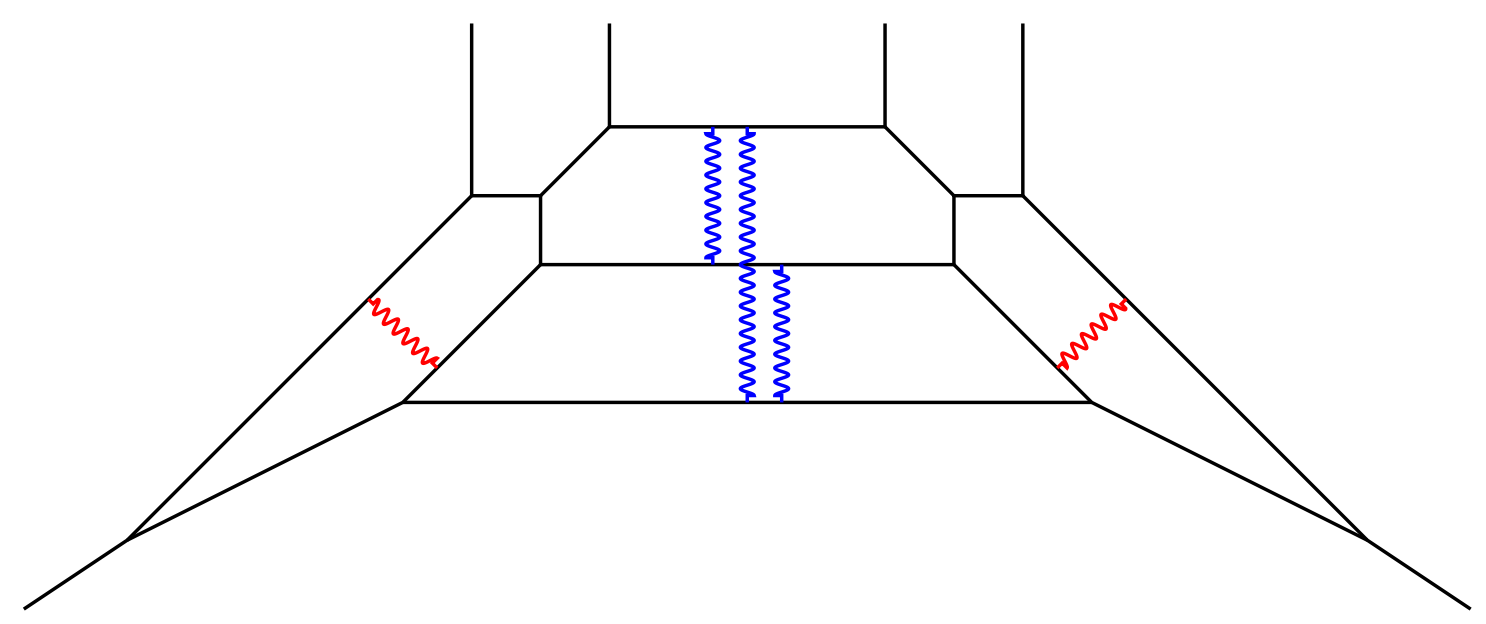

where the colored wavy lines indicate strings between parallel five-branes which generate the $S U(3) \times S U(2) \times S U(2)$ non-abelian gauge symmetry. Altogether, the model has 3 nondynamical coupling/mass parameters and 4 dynamical moduli, but their roles are mixed up: One of the two moduli controlling the $S U(3)$ breaking also controls the gauge couplings of the two $S U(2)$ factors. Consequently, one cannot un-Higgs the entire $S U(3) \times S U(2) \times S U(2)$ 
symmetry while keeping all the gauge couplings finite.

We have other examples of weird $5 \mathrm{D}$ theories following from $\mathrm{M}$ theory orbifolds $\mathbb{C}^{3} / \mathbb{Z}_{N}$, and we hope to present the whole menagerie in a future publication. For the moment, we return to the main subject of this article - the $S U(M)$ SYM theories in $5 \mathrm{D}$ and their moduli/parameter spaces. We have learned how everything works in the M/string based UV completions of the 5D SYM, so let us consider the alternative UV completion via dimensional deconstruction.

\section{Deconstruction}

In this section, we use dimensional deconstruction to study the quantum moduli and parameter spaces of the 5D $S U(M)$ SYM theory. That is, we deconstruct the fifth dimension of the theory and replace it with a discrete lattice of small bit finite spacing $a$. This breaks the Lorentz symmetry down to $\operatorname{Spin}(3,1)$ and also breaks 4 out of 8 supercharges of the theory. The result is a $4 \mathrm{D}, \mathcal{N}=1$ gauge theory on the following quiver:

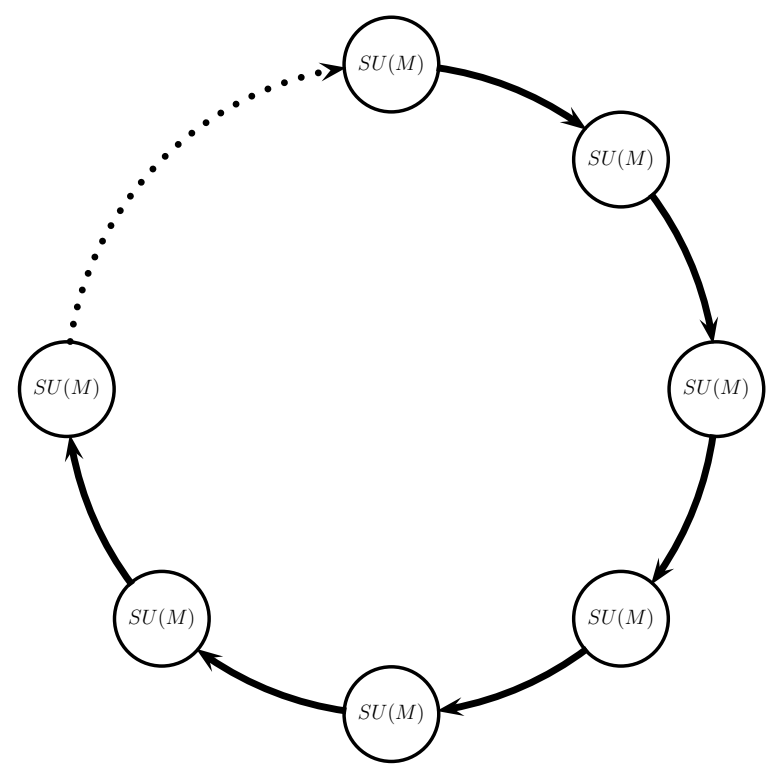

That is, the $4 \mathrm{D}$ gauge symmetry is

$$
G_{4}=\prod_{\ell=1}^{N} S U(M)_{\ell}
$$


while the chiral superfields comprise $N$ bi-fundamental multiplets

$$
Q_{\ell}=\left(\mathbf{M}_{\ell}, \overline{\mathbf{M}}_{\ell+1}\right), \quad \ell=1,2, \ldots, N \text { modulo } N
$$

Classically, the scalars in $Q_{\ell}$ are related to the 5D fields according to

$$
Q_{\ell}=V \times\left[\mathbf{1}+a \times\left(\phi+i A^{5}\right)_{x=\ell a}+O\left(a^{2} \phi^{2}\right)\right]
$$

for some fixed $V \neq 0$.

In a vacuum state $\left(\phi+i A^{5}=\right.$ const modulo gauge equivalence, $\left.\left[\phi, A^{5}\right]=0\right)$, the gauge symmetry (49) is spontaneously broken down to the diagonal $S U(M)$ (for $\phi+i A^{5}=0$ ) or a subgroup thereof; for generic $\phi+i A^{5}$, only the diagonal Cartan subgroup $U(1)^{M-1} \subset$ $S U(M)_{\text {diag }}$ survives unbroken. All the remaining vector fields acquire tree-level masses

$M^{2}\left(W_{k}^{i j}\right)=g^{2}|V|^{2}(1+O(a \phi)) \times\left[4 \sin ^{2} \frac{1}{2}\left(\frac{2 \pi k}{N}+a\left(A_{i}^{5}-A_{j}^{5}\right)\right)+\left(\phi_{i}-\phi_{j}\right)^{2} a^{2}(1+O(a \phi))\right]$

where $i$ and $j$ are $S U(M)$ indices (in the eigenbasis of $\phi$ and $A^{5}$ ) and $k$ is discrete Fourier transform of the quiver index $\ell$. Let us identify [15, 16]

$$
g^{2}|V|^{2}=\frac{1}{a^{2}}
$$

then for weak fields $\phi \ll(1 / a)$ and low 5 -momenta $k \ll N$, the spectrum (52) becomes exactly the Kaluza-Klein spectrum

$$
M^{2}=\left(\phi_{i}-\phi_{j}\right)^{2}+p_{5}^{2}, \quad p_{5}=\frac{2 \pi k}{N a}+\left(A_{i}^{5}-A_{j}^{5}\right)
$$

of the Coulomb branch of the 5D theory compactified on a circle of length $L=N a$. In the large quiver limit $N \rightarrow \infty$, the fifth dimension un-compactifies and the spectrum becomes continuous. 


\subsection{Deconstructing the Gauge Couplings}

In the quantum theory of the quiver (48), the masses (52) are corrected by all kinds of perturbative and non-perturbative effects, and we do not have any tools for deriving an exact formula. Instead, we focus on the holomorphic gauge couplings $\tau_{i j}$ of the unbroken $4 \mathrm{D}$ gauge symmetry $U(1)^{M-1}$. We shall calculate these couplings exactly and show that in the large quiver limit $N \rightarrow \infty$ they behave as

$$
2 \pi \operatorname{Im} \tau_{i j} \equiv\left[\frac{8 \pi^{2}}{g_{4}^{2}}\right]_{i j}=N a \times\left[\frac{8 \pi^{2}}{g_{5}^{2}}\right]_{i j}+O(1),
$$

in complete agreement with the Kaluza-Klein reduction of a 5D gauge theory. Furthermore, the 5D gauge coupling matrix on the right hand side of eq. (55) are exactly as in eq. (5) for the un-deconstructed 5D $S U(M)$ SYM.

Our first step is to relate the 5D eigenvalues $\phi_{i}$ to the holomorphic gauge-invariant moduli of the quiver theory. Altogether, the Coulomb branch of the quiver has

$$
\text { \#(Chiral SF })-\#(\text { Broken Symmetries })=N+M-1
$$

independent moduli, for example the "baryon" fields $B_{\ell}=\operatorname{det}\left(Q_{\ell}\right)$, and the coefficients $T_{1}, T_{2}, \ldots, T_{M-1}$ of the characteristic polynomial

$$
\begin{aligned}
T(X) & \equiv \operatorname{Det}\left(X-Q_{1} Q_{2} \cdots Q_{N}\right) \\
& =X^{M}-T_{1} X^{M-1}+T_{2} X^{M-2}-\cdots \mp T_{M-1} X \pm T_{M}
\end{aligned}
$$

of the quiver-ordered matrix product of the bilinear fields $Q_{\ell}$. The classical relation (51) between the $Q_{\ell}$ and the $5 \mathrm{D}$ fields yields $B_{\ell}=V^{M}$ and

$$
\begin{aligned}
T(X) & =\prod_{i=1}^{M}\left(X-V^{N} \exp \left(N a \varphi_{i}\right)\right) \\
\text { where } \varphi_{i} & =\phi_{i}+i A_{i}^{5}+O\left(a \phi^{2}\right) .
\end{aligned}
$$

Consequently, in the quantum quiver theory, we define

$$
\varphi_{i} \equiv \phi_{i}+i A_{i}^{5} \equiv \frac{1}{N a} \log \frac{R_{i}}{V^{N}}
$$


where $R_{i}$ are the roots of the characteristic polynomial $T(X)=\prod_{i}\left(X-R_{i}\right)$. In order to assure $\sum_{i} \varphi_{i}=0$ as required by the $S U(M)$ theory, we define $V$ according to

$$
V^{M N}=\prod_{i=1}^{M} R_{i}=T_{M}
$$

Note that $T_{M}$ is not an independent modulus of the quiver but a function of the baryon moduli $B_{\ell}$ [17, 18. Classically,

$$
T_{M} \equiv\left\langle\operatorname{Det}\left(Q_{1} Q_{2} \cdots Q_{N}\right)\right\rangle=\prod_{\ell=1}^{N}\left(\left\langle Q_{\ell}\right\rangle \equiv B_{\ell}\right)
$$

but quantum corrections [20, 21] modify this relation to

$$
\begin{aligned}
T_{M}= & \text { Polynomial part of }\left[\prod_{\ell} B_{\ell} \times \prod_{\ell}\left(1+\frac{\Lambda_{\ell}^{2 M}}{B_{\ell} B_{\ell+1}}\right)\right] \\
= & \prod_{\ell} B_{\ell} \times\left\{\begin{array}{c}
1+\sum_{\ell} \frac{\Lambda_{\ell}^{2 M}}{B_{\ell} B_{\ell+1}}+\frac{1}{2} \sum_{\ell} \sum_{\ell^{\prime} \neq \ell, \ell \pm 1} \frac{\Lambda_{\ell}^{2 M}}{B_{\ell} B_{\ell+1}} \frac{\Lambda_{\ell^{\prime}}^{2 M}}{B_{\ell^{\prime}} B_{k \ell^{\prime}+1}} \\
+\frac{1}{6} \sum_{\ell} \sum_{\ell^{\prime} \neq \ell, \ell^{\prime} \pm 1} \sum_{\ell^{\prime \prime} \neq \ell, \ell \pm 1, \ell^{\prime}, \ell^{\prime} \pm 1} \frac{\Lambda_{\ell}^{2 M}}{B_{\ell} B_{\ell+1}} \frac{\Lambda_{\ell^{\prime}}^{2 M}}{B_{\ell^{\prime}} B_{\ell^{\prime}+1}} \frac{\Lambda_{\ell^{\prime \prime}}^{2 M}}{B_{\ell^{\prime \prime}} B_{\ell^{\prime \prime}+1}}
\end{array}\right\}
\end{aligned}
$$

For the deconstruction purposes we are interested in translationally $\left(\mathbb{Z}_{N}\right)$ invariant quivers with equal $\Lambda_{\ell} \equiv \Lambda$. Likewise, we fix the baryon moduli to equal values $B_{\ell} \equiv B$.f Conse-

\footnotetext{
${ }^{4}$ The simplest way to fix all the baryonic moduli is to endow the quiver with a tree-level superpotential of the form

$$
W=\sum_{\ell}\left(\operatorname{det}\left(Q_{\ell}\right)-B\right) \times S_{\ell}
$$

where $S_{\ell}$ are some additional gauge-singlet chiral superfields. Although this superpotential is nonrenormalizable for $M>2$, the resulting divergences of the $4 \mathrm{D}$ theory can be regularized using covariant higher-derivative Lagrangian terms without affecting any of the holomorphic properties of the quiver.

Alternatively, we may leave the baryons un-fixed and gauge the $U(1)^{N}$ flavor symmetry of the quiver. From the 5D point of view, this would mean converting a non-dynamical parameter $h$ of the prepotential (ब) into a dynamical vector multiplet.
} 
quently, eq. (63) yields

$$
T_{M}=\sum_{n=0}^{\text {int }[N / 2]} \frac{N(N-n-1) !}{n !(N-2 n) !} B^{N-2 n} \Lambda^{2 M n},
$$

which sums to

$$
T_{M}=v_{1}^{M N}+v_{2}^{M N}
$$

where $v_{1,2}^{M}=\frac{1}{2}\left(B \pm \sqrt{B^{2}+4 \Lambda^{2 M}}\right)$ are the two roots of the quadratic equation system

$$
v_{1}^{M}+v_{2}^{M}=B, \quad v_{1}^{M} \times v_{2}^{M}=\Lambda^{2 M} .
$$

In the large quiver limit $N \rightarrow \infty$, the right hand side of eq. (66) is completely dominated by the larger of the two terms there, hence

$$
\lim _{N \rightarrow \infty} T_{M}=\max \left(v_{1}^{M N}, v_{2}^{M N}\right)
$$

and in light of eq. (60),

$$
V=\max \left(v_{1}, v_{2}\right)
$$

Note that according to eqs. (67) and (69),

$$
|V| \geq|\Lambda|
$$

regardless of the baryon VEV $B$. Although $V$ is a holomorphic parameter of the quiver, in the large $N$ limit there is a discontinuity which keeps $V$ outside a complex circle of radius $|\Lambda|$, and any attempt to analytically continue $V$ inside that circle results in a root exchange $v_{1} \leftrightarrow v_{2}$ and hence inversion $V \rightarrow \Lambda^{2} / V$ back to the outside of the $|\Lambda|$ circle.

Now that we understand the moduli of the quiver theory, let us consider the moduli dependence of the gauge couplings of its Coulomb branch. Logically, the gauge symmetry of the quiver is broken in two stages,

$$
[S U(M)]^{N} \rightarrow S U(M)_{\operatorname{diag}} \rightarrow U(1)^{M-1}
$$

where the second stage breaking is due to an effective adjoint multiplet of the diagonal $S U(M)$. Consequently, the quiver has a Seiberg-Witten hyperelliptic curve similar to that 
of an $S U(M)$ theory with an adjoint matter multiplet $A$, namely

$$
Y^{2}=[\operatorname{Det}(X-A)]^{2}-4 \Lambda^{2 M}
$$

For the quiver, the the role of the adjoint $A$ is played by the quiver-ordered product $Q_{1} Q_{2} \cdots Q_{N}$ of the bilinear fields while the $\Lambda^{2 M}$ of the diagonal $S U(M)$ becomes $\prod_{\ell} \Lambda_{\ell}^{2 M}$, hence the Seiberg-Witten curve

$$
\begin{aligned}
Y^{2} & =[T(X)]^{2}-4 \Lambda^{2 M N} \\
& =\prod_{i=1}^{M}\left(X-V^{N} e^{N a \varphi_{i}}\right)^{2}-4 \Lambda^{2 M N}
\end{aligned}
$$

We may eliminate the $V^{N}$ parameter from this formula by rescaling other variables. Let

$$
\begin{aligned}
x & =\frac{X}{V^{N}}, \quad y=\frac{Y}{V^{M N}}, \\
r_{i} & =\frac{R_{i}}{V^{N}}=\exp \left(N a \varphi_{i}\right), \\
t(x) & =\frac{T(X)}{V^{M N}}=\prod_{i=1}^{M}\left(x-r_{i}\right), \\
\lambda & =\frac{\Lambda^{M}}{V^{M}} ;
\end{aligned}
$$

then the rescaled Seiberg-Witten curve of the quiver becomes simply

$$
y^{2}=[t(x)]^{2}-4 \lambda^{2 N}
$$

Thanks to the bound (70), $|\lambda| \leq 1$; generically $|\lambda|<1$ and hence $\lambda^{2 N} \ll 1$ in the large quiver limit $N \rightarrow \infty$. Consequently, the $2 M$ branching points of the hyperelliptic curve (75) come in close pairs

$$
y=0, \quad x=r_{i, \pm}=r_{i} \pm \Delta_{i}
$$

where

$$
\Delta_{i}=\frac{2 \lambda^{N}}{t^{\prime}\left(x=r_{i}\right)} \ll\left|r_{i}-r_{j}\right| \forall j \neq i
$$

provided all the eigenvalues $\phi_{i}=\operatorname{Re} \varphi$ are distinct. That is, we focus on generic points of the Coulomb branch moduli space where all the non-abelian symmetries are broken. To avoid 
finite-size effects, we assume

$$
\forall i \neq j \quad\left|\phi_{i}-\phi_{j}\right| \gg \frac{1}{N a}
$$

hence

$$
r_{1} \ll r_{2} \ll r_{3} \ll \cdots \ll r_{M}
$$

(assuming the eigenvalues are ordered according to eq. (2)) and therefore

$$
\begin{aligned}
t^{\prime}\left(x=r_{i}\right) & =\prod_{j \neq i}\left(r_{i}-r_{j}\right) \approx\left(r_{i}\right)^{i-1} \times \prod_{j=i+1}^{M}\left(-r_{j}\right) \\
& =\frac{ \pm 1}{r_{i}} \times \prod_{j=i+1}^{M} \frac{r_{i}}{r_{j}} \gg \frac{1}{r_{i}},
\end{aligned}
$$

where the last equality follows from $\sum_{j=1}^{M} \varphi_{j}=0 \Rightarrow \prod_{j=1}^{M} r_{j}=1$. Consequently,

$$
\Delta_{i} \approx \pm 2 r_{i} \lambda^{N} \times \prod_{j<i} \frac{r_{j}}{r_{i}} \ll r_{i} \lambda^{N} \ll r_{i}
$$

and therefore indeed $\Delta_{i} \ll\left(r_{i}-r_{j}\right) \forall j \neq i$.

In the Appendix to this article, we show that a hyperelliptic Seiberg-Witten curve whose branching points come in closed pairs as in eq. (76) yields weak gauge couplings. Specifically, the holomorphic coupling matrix $\tau_{i j}$ is given by

$$
\begin{aligned}
\tau_{i \neq j} & =\frac{i}{2 \pi} \log \frac{r_{i} r_{j}}{\left(r_{i}-r_{j}\right)^{2}}, \\
\tau_{i=j} & =\frac{i}{2 \pi} \log \frac{4 r_{i}^{2}}{\Delta_{i}^{2}} .
\end{aligned}
$$

For the problem at hand,

$$
\frac{r_{i} r_{j}}{\left(r_{i}-r_{j}\right)^{2}} \approx \min \left(\frac{r_{i}}{r_{j}}, \frac{r_{j}}{r_{i}}\right)=\exp \left( \pm N a\left(\varphi_{i}-\varphi_{j}\right)\right)
$$

hence for $i \neq j$

$$
\tau_{i j}=\frac{i N a}{2 \pi} \times \begin{cases}\left(\varphi_{i}-\varphi_{j}\right) & \text { for } i<j \\ \left(\varphi_{j}-\varphi_{i}\right) & \text { for } j<i\end{cases}
$$


At the same time,

$$
\frac{4 r_{i}^{2}}{\Delta_{i}^{2}}=\lambda^{-2 N} \times \prod_{k<i} \exp \left(2 N a\left(\varphi_{i}-\varphi_{k}\right)\right)
$$

and hence

$$
\tau_{i i}=\frac{i N a}{2 \pi} \times\left[H+2 \sum_{k<i}\left(\varphi_{i}-\varphi_{k}\right)\right]
$$

where

$$
H=\frac{1}{a} \log \frac{1}{\lambda^{2}}=\frac{1}{a} \log \frac{V^{2 M}}{\Lambda^{2 M}}
$$

Note that (in the large quiver limit $N \rightarrow \infty$ ) the entire gauge coupling matrix $\tau_{i j}$ is proportional to the total length $L=N a$ of the deconstructed fifth dimension, in perfect agreement with the Kaluza-Klein reduction. Furthermore, by reversing the KK reduction (55) we immediately obtain the 5D gauge couplings of the deconstructed theory,

$$
\begin{aligned}
{\left[\frac{8 \pi^{2}}{g_{5}^{2}}\right]_{i j} } & =\delta_{i j} \times\left[\operatorname{Re} H+2 \sum_{k<i}\left(\phi_{i}-\phi_{k}\right)\right]-\left|\phi_{i}-\phi_{j}\right| \\
& =\delta_{i j} \times\left[\operatorname{Re} H+M \phi_{i}+\sum_{k=1}^{M}\left|\phi_{i}-\phi_{k}\right|\right]-\left|\phi_{i}-\phi_{j}\right|
\end{aligned}
$$

As promised, these couplings are exactly as in eq. (5) for the special case of Chern-Simons level $k_{\mathrm{cs}}=M$, provided we identify the tree-level 5D inverse gauge coupling $h$ with $\operatorname{Re} H$,

$$
h \equiv\left[\frac{8 \pi^{2}}{g_{5}^{2}(S U(M)}\right]^{\text {tree }}=\operatorname{Re} H=\frac{1}{a} \log \left|\frac{V}{\Lambda}\right|^{2 M}
$$

Thanks to the bound (70), $h \geq 0$ throughout the entire moduli space of the quiver and there are no "flop" transitions to other phases of the 5D theory. In other words, we have deconstructed the coupling parameter space of the quantum 5D SYM theory as the positive real semi-axis $\mathbb{R}^{+}$, in perfect agreement with the $M$-theory results for $k_{\mathrm{cs}}=M$.

From the 5D SYM point of view, deconstructing the fifth dimension serves as a UV completion of the theory in a radically different way that the M-theory embeddings and the $(p, q)$ webs discussed in section 2. Yet for all their differences, we end up with exactly the same parameter space of the theory - $h \geq 0$ only, no flop transitions - which strongly 
suggests that this parameter space is the inherent property of the 5D SYM regardless of the UV completion one might want to use to work it out.

Note that the $\mathbb{R}^{+}$parameter space space without any flops is peculiar to the maximal Chern-Simons level $k_{\mathrm{cs}}=M$ of the 5D theory. As we saw in the previous section, a $5 \mathrm{D}$ SYM at a lower Chern-Simons level $k_{\mathrm{cs}}<M$ - or rather its UV completion via IIB $(p, q)$

5 -brane webs - does have a flop transition at $h=0$ from the ordinary SYM phase at $h>0$ to something more exotic at $h<0$. Again, this turns out to be an inherent property on the $5 \mathrm{D}$ theory, with exactly the same phases and flop transitions in the deconstructed theory as in the M-theory based completions.

Unfortunately, deconstructing the 5D theories with $k_{\mathrm{cs}}<M$ requires more complicated quivers than (48), so the authors prefer to present this issue in a separate follow-up article [14. We will show in detail how to deconstruct the 5D SQCD — with or without flavors - for all allowed Chern-Simons numbers, but our main conclusion can be stated right now: The deconstructed 5D SQCD has precisely the same 5D phases and flop transitions as the $M$-theory based UV completions of the same 5D theory. We emphasize the 5D phases here because some quiver theories also have additional, strongly-coupled phases - completely outside the 5D phase spaces — where the 4D gauge couplings are stuck at strong values, and the deconstruction does not seem to work. We suspect that such strongly-coupled 4D phases are related to superconformal theories in 5D, but further research is needed before we can be more definite on this issue. But the ordinary, weakly-coupled 4D phases of the deconstructed theory are precisely as in the M-based completions of the same 5D theory.

\subsection{Deconstructing Chern Simons}

In collaboration with Edoardo Di Napoli

It is well known that in a 5D gauge theory with 8 unbroken supercharges, the moduli dependence of the gauge couplings is related to the topological Chern-Simons couplings of the gauge fields, $c f$. eq. (3). But it isn't known yet whether this relation survives breaking of 4 out of 8 supercharges in the process of dimensional deconstruction. For example, the quiver (48) we used to deconstruct the 5D $S U(M)$ SYM theory yielded gauge couplings corresponding to the Chern-Simons level $k_{\mathrm{cs}}=M$, and the $\mathbb{R}^{+}$geometry of the parameter 
space of the theory gives further evidence for this identification. But because of the partial SUSY breakdown, we cannot be sure about the actual Chern-Simons interactions of between the 5D gauge fields of the deconstructed theory, and the only way to be sure is to derive them directly from the quiver theory.

In this subsection, we shall see that the deconstructed theory indeed has Chern-Simons action

$$
S_{\mathrm{CS}}=k_{\mathrm{cs}} \times \int_{\mathbb{R}^{5}} \Omega_{\mathrm{CS}}
$$

with the correct coefficient $k_{\mathrm{cs}}=M$. To stress the tree-level nature of these interactions from the 5D point of view, we shall assume un-broken $S U(M)$ symmetry in 5D and look for the non-abelian Chern-Simons 5-form

$$
\Omega_{\mathrm{CS}}=\frac{i}{24 \pi^{2}} \operatorname{tr}\left(A \wedge F \wedge F-\frac{i}{2} A \wedge A \wedge A \wedge F-\frac{1}{10} A \wedge A \wedge A \wedge A \wedge A\right) .
$$

Thus, we assume $\langle\phi\rangle=0$ and no Wilson lines, or in quiver terms,

$$
\left\langle Q_{\ell}\right\rangle=V U_{\ell}, \quad \text { unitary } U_{\ell}=\left(U_{\ell}^{\dagger}\right)^{-1}, \quad U_{1} U_{2} \cdots U_{N}=1 .
$$

Likewise, in order to avoid unwarranted extended-SUSY based assumptions we shall work in the component-field formalism where $\psi_{\ell}$ are the $\mathcal{N}=1$ fermionic superpartners of the $Q_{\ell}$ scalars and $\lambda_{\ell}$ are the $\mathcal{N}=1$ gaugino fields of the $S U(M)_{\ell}$. The Yukawa couplings of these fermions follow from the unbroken SUSY of the quiver theory: In $M \times M$ matrix notations,

$$
\mathcal{L}_{Y}=\sum_{\ell} \operatorname{tr}\left(-i g Q_{\ell}^{\dagger} \lambda_{\ell} \psi_{\ell}+i g \psi_{\ell} \lambda_{\ell+1} Q_{\ell}^{\dagger}\right)+\text { H.c. }
$$

Given the scalar VEVs (91), these Yukawa couplings produce 4D fermionic mass terms with simple fixed eigenvalues but not-so-simple $U_{\ell}$ dependent mass eigenstates: Let

$$
\tilde{\psi}_{\ell}=\left(U_{1} U_{2} \cdots U_{\ell-1}\right)^{\dagger} \psi_{\ell}\left(U_{1} U_{2} \cdots U_{\ell-1} U_{\ell}\right), \quad \tilde{\lambda}_{\ell}=\left(U_{1} U_{2} \cdots U_{\ell-1}\right)^{\dagger} \lambda_{\ell}\left(U_{1} U_{2} \cdots U_{\ell-1}\right),
$$

then the fermionic mass terms become

$$
\mathcal{L}_{\text {masses }}^{\text {fermion }}=\left\{i g V \sum_{\ell} \operatorname{tr}\left(-\tilde{\lambda}_{\ell} \tilde{\psi}_{\ell}+\tilde{\psi}_{\ell} \tilde{\lambda}_{\ell+1}\right)=\sum_{\ell} i \tilde{\psi}_{\ell} \frac{\tilde{\lambda}_{\ell+1}-\tilde{\lambda}_{\ell}}{a}\right\}+\text { H.c. }
$$


which obviously deconstructs the fifth component of the 5D fermion's kinetic energy term. Indeed, combining $\tilde{\psi}_{\ell}$ with $\tilde{\bar{\lambda}}_{\ell}$ into a single 4 -component 5D Dirac spinor $\Psi_{\ell}$, we have

$$
\sum_{\ell} i \tilde{\psi}_{\ell} \frac{\tilde{\lambda}_{\ell+1}-\tilde{\lambda}_{\ell}}{a}+\text { H.c. }=\sum_{\ell} i \bar{\Psi}_{\ell} \gamma^{5} \frac{\Psi_{\ell+1}-\Psi_{\ell}}{a} \rightarrow \int d x^{5} i \bar{\Psi} \gamma^{5} \partial_{5} \Psi \text {. }
$$

Note $U_{\ell}$ dependent chiral rotation (93) involved in converting the original fermionic fields $\psi_{\ell}$ and $\lambda_{\ell}$ of the quiver into the components $\tilde{\psi}_{\ell}$ and $\tilde{\lambda}_{\ell}$ (or rather $\tilde{\bar{\lambda}}_{\ell}$ ) of the 5D fermion $\Psi_{\ell}$. Unless all the $U_{\ell}$ matrices are constant with respect to 4 continuous dimensions of spacetime, the transform (93) has a non-trivial functional determinant, which gives rise to the WessZumino terms for the $U_{\ell}(x)$ at the one-loop level of the perturbation theory.

By way of analogy, consider the Wess-Zumino terms on the ordinary QCD with $n_{c}>2$ colors and $n_{f}>2$ flavors. The spontaneous breakdown $S U\left(n_{f}\right)_{L} \times S U\left(n_{f}\right)_{R} \rightarrow S U\left(n_{f}\right) V$ of the chiral symmetry of the theory gives rise to an effective non-linear sigma model of the matrix field $U(x) \in S U\left(n_{f}\right)$. It also gives rise to a chiral transform of the original fermionic fields of the theory - the so-called current quark and antiquark fields - into the eigenstates of the mass matrix known as the constituent quarks. In terms of left-handed quarks $\psi$ and antiquarks $\chi$, the transform works as

$$
\psi^{\prime}(x)=\psi(x) \times W(x) U(x), \quad \chi^{\prime}(x)=\chi(x) \times W(x)
$$

for some arbitrary vector-like flavor symmetry $W(x) \in S U\left(n_{f}\right)_{V}$, but the functional determinant depends only on the chiral rotation $U(x)$. The logarithm of this functional determinant is the Wess-Zumino action term for the pseudoscalar meson fields comprising the $U$ matrix,

$$
S_{\mathrm{WZ}}=\log \operatorname{Det}(\psi(x) \rightarrow \psi(x) U(x))=n_{c} \times \int_{\mathbb{R}^{4}} \Omega_{\mathrm{WZ}}[U]
$$

where the $n_{c}$ factor reflect $n_{c}$ identical multiplets of the chiral flavor symmetry suffering the same chiral rotation $U(x)$. The basic Wess-Zumino 4 -form $\Omega_{\mathrm{WZ}}$ is ugly and lacks manifest 
$S U\left(n_{f}\right) \times S U\left(n_{f}\right)$ symmetry, but its formal exterior derivative is nice and symmetric

$$
d \Omega_{\mathrm{WZ}}[U]=\frac{i}{240 \pi^{2}} \operatorname{tr}(J \wedge J \wedge J \wedge J \wedge J) \quad \text { where } J_{\mu}=-i\left(\partial_{\mu} U\right) U^{\dagger} .
$$

Therefore, the Wess-Zumino term is usually written as the 5D integral

$$
S_{\mathrm{WZ}}=n_{c} \int_{\mathcal{B}^{5}} d \Omega_{\mathrm{WZ}}[U]
$$

over a solid ball $\mathcal{B}^{5}$ whose surface $S^{4}$ is topologically identified with the Euclidean spacetime of the fermionic path integral. That is, we introduce an auxiliary fifth dimension $t$ of finite range $0 \leq t \leq 1$ and extrapolate

$$
\begin{aligned}
U\left(x^{1}, x^{2}, x^{3}, x^{4}\right) \mapsto & U\left(x^{1}, x^{2}, x^{3}, x^{4}, t\right) \in S U\left(n_{f}\right), \\
& U\left(x^{1}, x^{2}, x^{3}, x^{4}, t=1\right)=U\left(x^{1}, x^{2}, x^{3}, x^{4}\right), \\
& U\left(x^{1}, x^{2}, x^{3}, x^{4}, t=0\right) \equiv 1 .
\end{aligned}
$$

Consequently, $t$ acts as a radial coordinate of a 5 -ball $\mathcal{B}^{5}$ while the Euclidean spacetime coordinates $\left(x^{1}, x^{2}, x^{3}, x^{4}\right)$ act as its angular coordinatesff $J_{\mu}(\mu=1,2,3,4, t)$ is a matrixvalued 1 -form on this 5 -ball, and $d \Omega_{\mathrm{WZ}}$ of eq. (98) is a 5 -form.

In QCD, spontaneous breakdown of the chiral symmetry is a highly non-perturbative dynamical effect. However, once it happens, the Wess-Zumino term for the meson fields comprising $U(x)$ arises as a one-loop anomaly of the chiral transformation of the chiral fields. After this transform, one may leave the constituent quark fields as a part of the effective low-energy theory or integrate them out altogether: One way or the other, the Wess-Zumino terms remain a part of the meson field action.

In the quiver theory, the $[S U(M)]^{N}$ symmetry is spontaneously broken down (or rather Higgsed down) at the tree level rather than dynamically, but the effect on the fermionic fields of the theory is rather similar: The original fermions $\psi_{\ell}$ and $\lambda_{\ell}$ of the quiver suffer a $U_{\ell}$ dependent chiral transform (93) into mass eigenstates which deconstruct the 5D fermion

\footnotetext{
${ }^{5}$ We assume a well-defined limit $U(x \rightarrow \infty)$ as $x$ goes to infinity in any direction in the Euclidean space. Topologically, this makes the Euclidean space a sphere $S^{4}$.
} 
$\Psi$. Chirally speaking, the transform (93) combines a complicated but vector-like transform (analogous to the $W(x)$ matrix in eq. (96)) with a simple but chiral transform

$$
\psi_{\ell}(x) \rightarrow \psi_{\ell}(x) U_{\ell}(x), \quad \lambda_{\ell}(x) \rightarrow \lambda_{\ell}(x)
$$

whose functional determinant gives rise to the QCD-like Wess-Zumino action

$$
S_{\mathrm{WZ}}=\sum_{\ell} k_{\mathrm{wZ}} \int_{\mathcal{B}^{5}} d \Omega_{\mathrm{WZ}}\left(U_{\ell}\right)
$$

whose coefficient follows from the multiplicity of the "quark" fields $\psi_{\ell}$ : As far as the $U_{\ell}$ is concerned, $\psi_{\ell}$ comprises $M$ "colors" undergoing similar chiral transforms, hence

$$
k_{\mathrm{wz}}=M
$$

From the 5D point of view, the Wess-Zumino terms (102) deconstruct the 5D ChernSimons action (89) [22, 23]. To see how this works, we need to consider not just five but six spacetime dimensions: The four continuous dimensions $x^{1}, \ldots x^{4}$ of the quiver theory, the deconstructed fifth dimension $x^{5}=a \ell$ discretized via quiver label $\ell$, and finally the auxiliary dimension $t$ of the Wess-Zumino action. Please note that although both the $x^{5}$ and the $t$ are often called "the fifth dimension," they are quite distinct and should not be confused with each other. In eq. (102), the 5 -ball $\mathcal{B}^{5}$ spans $\left(x^{1}, x^{2}, x^{3}, x^{4}, t\right)$ while the $x^{5}$ dimension is discretized as $\ell$.

We must also modify eq. (98) for the Wess-Zumino 5-form to account for the gauging of the $S U(M)_{\ell} \times S U(M)_{\ell+1}$ "flavor" symmetry of each $U_{\ell}$. Altogether, the Wess-Zumino action comes to

$$
S_{\mathrm{WZ}}=\sum_{\ell} \frac{i k_{\mathrm{wz}}}{240 \pi^{2}} \int_{\mathcal{B}^{5}} \operatorname{tr}\left\{\begin{array}{c}
J \wedge J \wedge J \wedge J \wedge J-5 i J \wedge J \wedge J \wedge(F+H) \\
+5 J \wedge(2 F \wedge F+2 H \wedge H+F \wedge H+H \wedge F)
\end{array}\right\}_{\ell}
$$


where

$$
\begin{aligned}
J_{\ell} & =-i D U_{\ell} U_{\ell}^{\dagger}=-i d U_{\ell} U_{\ell}^{\dagger}+A_{\ell}-U_{\ell} A_{\ell+1} U_{\ell}^{\dagger} \\
D J_{\ell} & =i J_{\ell} \wedge J_{\ell}+F_{\ell}-H_{\ell} \\
F_{\ell} & =d A_{\ell}+i A_{\ell} \wedge A_{\ell} \\
H_{\ell} & =U_{\ell} F_{\ell+1} U_{\ell}^{\dagger}
\end{aligned}
$$

and the gauge-anomaly terms contained in each $d \Omega_{\mathrm{CS}}\left[U_{\ell}\right]$ cancel out of the $\sum_{\ell}$. The action (104) is valid for arbitrary $S U(M)$ matrices $U_{\ell}(x)$, but for the deconstruction purposes we are interested in the continuous $x^{5}$ limit $a \rightarrow 0$ where

$$
U_{\ell}=1+i a A_{5}+O\left(a^{2}\right) \text { at } x^{5}=a \ell
$$

cf. eq. (51). In this limit $H_{\ell}=F_{\ell}+O(a)$ while

$$
J_{\ell, \mu}=a\left(\partial_{\mu} A_{5}-\partial_{5} A_{\mu}+i\left[A_{\mu}, A_{5}\right]\right)+O\left(a^{2}\right) \equiv a F_{\mu 5}+O\left(a^{2}\right),
$$

or in the 5D 1-form notations, $J_{\ell}=a F_{\ell}^{5}+O\left(a^{2}\right)$. Consequently, to the leading order in $a$,

$$
\begin{aligned}
S_{\mathrm{WZ}} & =\sum_{\ell} \frac{i k_{\mathrm{wz}}}{240 \pi^{2}} \int_{\mathcal{B}^{5}} \operatorname{tr}\left\{30 a F_{\ell}^{5} \wedge F_{\ell} \wedge F_{\ell}+O\left(a^{2}\right)\right\} \\
& \longrightarrow \frac{i k_{\mathrm{wz}}}{8 \pi^{2}} \int_{S^{1}} d x^{5} \int_{\mathcal{B}^{5}} \operatorname{tr}\left(F^{5} \wedge F \wedge F\right)_{5 \mathrm{D}} \\
& =\frac{i k_{\mathrm{wz}}}{24 \pi^{2}} \int_{S^{1} \times \mathcal{B}^{5}} \operatorname{tr}(F \wedge F \wedge F)_{6 \mathrm{D}} .
\end{aligned}
$$

But in six dimensions

$$
\frac{i}{24 \pi^{2}} \operatorname{tr}(F \wedge F \wedge F)=d \Omega_{\mathrm{CS}}
$$

is the total derivative of the Chern-Simons 5-form (90), hence in the continuum limit

$$
S_{\mathrm{WZ}} \underset{a \rightarrow 0}{\longrightarrow} k_{\mathrm{wZ}} \int_{S^{1} \times \mathcal{B}^{5}} d \Omega_{\mathrm{CS}}=k_{\mathrm{wZ}} \int_{S^{1} \times S^{4}} \Omega_{\mathrm{CS}}
$$

where the deconstructed fifth dimension $x^{5}$ spans the circle $S^{1}$, the continuous spacetime dimensions $\left(x^{1}, x^{2}, x^{3}, x^{4}\right)$ are topologically compactified to $S^{4}$ and the auxiliary dimension 
$t$ has disappeared from the final formula. Apart from topological subtleties of the deconstructed 4D spacetime being $S^{1} \times S^{4}$ instead of $S^{5}$ or $\mathbb{R}^{5}$, the action (110) is precisely the Chern-Simons action (89) we wanted to deconstruct, with precisely the right coefficient

$$
k_{\mathrm{cs}}=k_{\mathrm{wz}}=M .
$$

This somewhat lengthy exercise in "topological deconstruction" verifies $k_{\mathrm{cs}}=M$ for the quiver (48), but it also helps us answer a deeper question, namely how does one deconstruct a $5 D S Y M$ with $k_{\mathrm{cs}} \neq M$ ? Clearly, the Wess-Zumino / Chern-Simons connection is rather rigid and does not allow any deviations from $k_{\mathrm{cs}}=k_{\mathrm{wz}}$. On the other hand, we can change the Wess-Zumino level of the quiver if and only if we change the fermionic fields or the chiral transforms they suffer while being packaged into the 5D fermions. Without breaking the $\mathcal{N}=1$ SUSY of the quiver theory, we cannot tinker with $\psi_{\ell}$ and the $\lambda_{\ell}$ fermions, but we may add more fermions to the theory. In the follow-up article [14] we shall use this approach do deconstruct the 5D SQCD — with or without flavors - at any allowed Chern-Simons level

$$
\left|k_{\mathrm{cs}}\right| \leq n_{c}-\frac{n_{f}}{2}, \quad k_{\mathrm{cs}} \in \mathbb{Z}+\frac{n_{f}}{2}
$$

\section{Acknowledgments}

The authors would like to thank Jacob Sonnenschein, Yaron Oz and Edoardo Di Napoli for valuable discussions. This research was supported by the US National Science Foundation (grants NSF-DMS/00-74329 and PHY-0071512), the Robert A. Welsh foundation and the US-Israel Bi-National Science Foundation. 


\section{Appendix: Hyperelliptic Seiberg-Witten Curves}

Consider an abelian rank $r$ Coulomb branch of a generic 4D supersymmetric gauge theory. The holomorphic gauge couplings of the abelian fields form an $r \times r$ symmetric matrix $\tau_{i j}$ defined modulo $S p(r, \mathbb{Z})$ symmetry group of electric-magnetic dualities. The dualityindependent representation of the coupling matrix is given by a hyperelliptic Seiberg-Witten curve of genus $g=r$, generally of the form

$$
y^{2}=\prod_{i=1}^{2 r+2}\left(x-b_{i}\right)
$$

where the branching points $b_{i}$ are distinct for generic values of the theory's moduli but collide when some charged particles become massless.

The first homology $H^{(1)}(\mathbb{Z})$ of the curve (113) has a symplectic product — the intersection number. Choosing a particular symplectic basis of 1 -cycles $\alpha_{1}, \ldots, \alpha_{r}, \beta_{1}, \ldots, \beta_{r}$,

$$
\alpha_{i} \cap \beta_{j}=\delta_{i j}, \quad \alpha \cap \alpha \equiv \beta \cap \beta \equiv 0,
$$

corresponds to choosing a basis of the electric and the magnetic charges and hence of the electric and the magnetic fields. In this basis, the coupling matrix $\tau_{i j}$ relates the periods of holomorphic 1-forms of the curve,

$$
\begin{aligned}
\oint_{\beta_{i}} \Omega & =\sum_{j} \tau_{i j} \times \oint_{\beta_{i}} \Omega, \\
\Omega & =\frac{d x}{y} \times \operatorname{Polynomial}(x) \text { of degree }(r-1) .
\end{aligned}
$$

In this Appendix we evaluate the couplings of hyperelliptic curves whose branching points come in very close pairs,

$$
\begin{aligned}
y^{2} & =\prod_{i=1}^{M}\left(x-r_{i,+}\right)\left(x-r_{i,-}\right), \\
r_{i, \pm} & =r_{i} \pm \Delta_{i}, \quad\left|\Delta_{i}\right| \ll\left|r_{i}-r_{j}\right| \forall j \neq i .
\end{aligned}
$$

Geometrically, such a curve looks like a pair of complex spheres connected by $M$ very thin 
tubes,

$$
\begin{array}{lc}
y \pm \pm \prod_{i=1}^{M}\left(x-r_{i}\right) & \text { for } x \text { away from all } r_{i} \\
& \text { i.e. }, \forall i:\left(x-r_{i}\right) \gg \Delta_{i}, \\
y \approx \pm \sqrt{\left(x-r_{i}\right)^{2}-\Delta_{i}^{2}} \times \prod_{j \neq i}\left(x-r_{j}\right) & \text { for } x \text { near } r_{i},
\end{array}
$$

which gives us a natural choice of the "electric" $\alpha$ cycles of the curve: $\alpha_{i}$ runs around the tube around $r_{i}$. Equivalently, we let the square-root branch cuts of the curve (117) connect each branching point $r_{i, \pm}$ to its closest neighbor $r_{i, \mp}$ and let the cycle $\alpha_{i}$ run around the cut between the $r_{i,-}$ and the $r_{i,+}$. Notice that homologically

$$
\alpha_{1}+\alpha_{2}+\cdots+\alpha_{M}=0
$$

and only $r=M-1$ of these $\alpha_{i}$ cycles are independent. This redundancy matches the redundancy of the abelian gauge field basis $A_{i}^{\mu}$ we use in the main body of this article: For the Coulomb branch of a 5D $S U(M)$ theory, the $A_{i}^{\mu}$ are superpartners of the scalar eigenvalues $\phi_{i}$, hence

$M$ vector fields $A_{1}^{\mu}, A_{2}^{\mu}, \ldots, A_{M}^{\mu}$ constrained to satisfy $A_{1}^{\mu}+A_{2}^{\mu}+\cdots+A_{M}^{\mu}=0$,

and we follow the same convention for the 4D quiver. Consequently, the corresponding $M \times M$ matrix $\tau_{i j}$ of the gauge couplings is redundant modulo

$$
\tau_{i j} \rightarrow \tau_{i j}^{\prime}=\tau_{i j}+c_{i}+c_{j} \quad \forall\left(c_{1}, c_{2}, \ldots, c_{M}\right)
$$

The "magnetic" $\beta$ cycles of the SW curve (117) run through (rather than around) the connecting tubes at $r_{i}$; two tubes are involved in each $\beta$ cycle. This gives us a very large set of magnetic cycles to choose from: For any $j \neq k$, we can set up the branch cuts of the curve such that the $r_{j,+}$ branching point is connected to the $r_{k,-}$; the cycle surrounding this cut (and no other cuts) we call $\beta_{j k}$. Naturally, only $M-1$ of these magnetic cycles are 
independent while the rest follow from the linear relations

$$
\beta_{i j}+\beta_{j k}=\beta_{i k}+\alpha_{j}
$$

Furthermore, the $\beta_{j k}$ cycles are not exactly dual to the electric cycles $\alpha_{i}$ : Instead of the symplectic intersections (114), we have

$$
\alpha \cap \alpha \equiv 0, \quad \beta \cap \beta \equiv 0, \quad \text { but } \quad \alpha_{i} \cap \beta_{j k}=\delta_{i j}-\delta_{i k}
$$

Consequently, the periods of holomorphic 1-forms of the SW curve are related to the gauge couplings according to

$$
\oint_{\beta_{j k}} \Omega=\sum_{i=1}^{M}\left(\tau_{i j}-\tau_{i k}+\text { an integer }\right) \times \oint_{\alpha_{i}} \Omega
$$

As usual, $\Omega=(d x / y) \times$ a polynomial of degree $M-2$ or less. To simplify the period calculations, we let

$$
\Omega=\frac{d x}{y} \times \sum_{i=1}^{M} C_{i} \prod_{j \neq i}\left(x-r_{j}\right), \quad \sum_{i=1}^{M} C_{i}=0 .
$$

hence in light of the curve geometry (119),

$$
\begin{array}{ll}
\Omega \approx \pm \sum_{i=1}^{M} \frac{C_{i} d x}{x-r_{i}} & \text { for } x \text { away from all } r_{i} \\
\Omega \approx \pm\left[\frac{C_{i} d x}{\sqrt{\left(x-r_{i}\right)^{2}-\Delta_{i}^{2}}}+\sum_{j \neq i} \frac{C_{j} d x}{x-r_{j}}\right] & \text { for } x \text { near } r_{i} \\
\Omega \approx \pm \sum_{i=1}^{M} \frac{C_{i} d x}{\sqrt{\left(x-r_{i}\right)^{2}-\Delta_{i}^{2}}} & \text { for } x \text { everywhere. }
\end{array}
$$

In terms of the $x$ coordinate, an electric cycle $\alpha_{i}$ is basically a small circle surrounding $r_{i} \pm \Delta_{i}$ 
hence the corresponding period of the $\Omega$ is simply

$$
\oint_{\alpha_{i}} \Omega \approx \oint_{\alpha_{i}} \frac{C_{i} d x}{\sqrt{\left(x-r_{i}\right)^{2}-\Delta_{i}^{2}}}+\sum_{j \neq i} \oint_{\alpha_{i}} \frac{C_{i} d x}{x-r_{i}}=2 \pi i C_{i}+0 .
$$

For a magnetic cycle $\beta_{j k}$, the integration contour runs from the $r_{j,+}$ to the $r_{k,-}$ on one Riemann sheet, then comes back on the other sheet where all the square roots take opposite signs. Hence, the magnetic period

$$
\begin{aligned}
\oint_{\beta_{j k}} \Omega & \approx-2 \int_{r_{j,+}}^{r_{k,-}} \sum_{i} \frac{C_{i} d x}{\sqrt{\left(x-r_{i}\right)^{2}-\Delta_{i}^{2}}}=-\left.2 \sum_{i} C_{i} \operatorname{arsinh} \frac{x-r_{i}}{\Delta_{i}}\right|_{r_{j,+}} ^{r_{k,-}} \\
& \approx 2\left[C_{j} \log \frac{\Delta_{j}}{2}+\sum_{i \neq j} C_{i} \log \left(r_{j}-r_{i}\right)\right]-2\left[C_{k} \log \frac{\Delta_{k}}{2}+\sum_{i \neq k} C_{i} \log \left(r_{j}-r_{k}\right)\right]
\end{aligned}
$$

Comparing these periods with eqs. (125), we immediately arrive at the gauge coupling matrix

$$
\tau_{i j}=\frac{2}{2 \pi i}\left\{\begin{array}{ll}
\log \left(r_{i}-r_{j}\right) & \text { for } i \neq j, \\
\log \frac{\Delta_{i}}{2} & \text { for } i=j,
\end{array}\right\}+O\left(\frac{\Delta_{i, j}^{2}}{\left(r_{i}-r_{j}\right)^{2}}\right)
$$

or equivalently — thanks to the redundancy (122),

$$
\begin{aligned}
\tau_{i \neq j} & =\frac{i}{2 \pi} \log \frac{r_{i} r_{j}}{\left(r_{i}-r_{j}\right)^{2}}, \\
\tau_{i i} & =\frac{i}{2 \pi} \log \frac{4 r_{i}^{2}}{\Delta_{i}^{2}} .
\end{aligned}
$$

Physically, the limit of very close pairs of branching points corresponds to the weak gauge couplings. Indeed, eqs. (131) show that in the $\Delta_{i} \rightarrow 0$ limit,

$$
g_{i}^{2} \approx \frac{4 \pi^{2}}{\log \left|r_{i} / \Delta_{i}\right|}+O\left(\frac{1}{\log ^{2}}\right) \rightarrow 0
$$

To get a more accurate picture, let us focus on the coupling of a particular abelian gauge 
field. For example, let $A_{i j}^{\mu}=A_{i}^{\mu}-A_{i}^{\mu}$; this field has coupling

$$
\begin{aligned}
\tau\left(A_{i j}^{\mu}\right) & =\tau_{i i}+\tau_{j j}-2 \tau_{i j} \\
& =\frac{i}{2 \pi} \log \frac{16\left(r_{i}-r_{j}\right)^{4}}{\Delta_{i}^{2} \Delta_{j}^{2}}+O\left(\left(\frac{\Delta^{2}}{\left(r_{i}-r_{j}\right)^{2}}\right)\right. \\
& =\frac{2 i}{2 \pi} \log \left(4 \chi_{i j}\right)+O\left(1 / \chi_{i j}\right)
\end{aligned}
$$

where

$$
\chi_{i j}=4 \frac{\left(r_{i,+}-r_{j,-}\right)\left(r_{i,-}-r_{j,+}\right)}{\left(r_{i,+}-r_{i,-}\right)\left(r_{j,+}-r_{j,-}\right)} \approx \frac{\left(r_{i}-r_{j}\right)^{2}}{\Delta_{i} \Delta_{j}}
$$

is the $S L(2, \mathbb{C})$ invariant cross-ratio of the 4 branching points $r_{i, \pm}$ and $r_{j, \pm}$. When the branching points come in closed pairs (118), all the $\chi_{i j}$ invariants are large, and all the gauge couplings are weak.

Note that the physical gauge couplings due to a Seiberg-Witten curve are invariant under the $S L(2, \mathbb{C})$ transforms of the complex $x$ plane. Hence, eqs. (133) remain valid as long as all $\chi_{i j} \gg 1$, even if some of the $r_{i, \pm}$ pairs are not close. The close-pairs condition (118) is sufficient to assure that all the physical gauge couplings are weak and calculable according to our formulae, but it is stronger than necessary.

In our next article [14] we will show that deconstructing 5D SQCD with flavors or with Chern-Simons levels $C<M$ leads to Seiberg-Witten curves which do not always satisfy the close-pairs condition (118). When a pair $r_{i, \pm}$ splits apart but all the cross-invariants $\chi_{i j}$ are large, the theory undergoes a flop transition in the large quiver limit. But when some of the cross-invariants become small, the low-energy 4D couplings become large and the deconstruction fails altogether. 


\section{References}

[1] A. Klemm, W. Lerche, P. Mayr, C. Vafa, N. Warner, "Self-Dual Strings and $N=2$ Supersymmetric Field Theory," Nucl. Phys. B477 (1996) 746-766, hep-th/9604034.

[2] E. Witten, "Solutions Of Four-Dimensional Field Theories Via M Theory," Nucl. Phys. B500 (1997) 3-42, hep-th/9703166.

[3] S. Katz, A. Klemm, C. Vafa, "Geometric Engineering of Quantum Field Theories," Nucl. Phys. B497 173-195, hep-th/9609239.

[4] Nathan Seiberg, "Five Dimensional SUSY Field Theories, Non-trivial Fixed Points and String Dynamics," hep-th/9608111.

[5] E. Witten, "Physical Interpretation of Certain Strong Coupling Singularities," Mod. Phys. Lett. A11 (1996) 2649-2654, hep-th/9609159.

[6] M. R. Douglas, S. Katz and C. Vafa, "Small Instantons, del Pezzo Surfaces and Type I' Theory," Nucl. Phys. B497 (1997) 155-172, hep-th/9609071.

[7] D. R. Morrison and N. Seiberg, "Extremal Transitions and Five Dimensional Supersymmetric Field Theories," Nucl. Phys. B483 (1997) 229, hep-th/9609070.

[8] K. Intriligator, D. R. Morrison and N. Seiberg, "Five-Dimensional Supersymmetric Gauge Theories and Degeneration of Calabi-Yau Spaces," Nucl. Phys. B497 (1997) 56, hep-th/9702198.

[9] O. Aharony, A. Hanany and B. Kol, "Webs of $(p, q)$ 5-Branes, Five Dimensional Field Theories and Grid Diagrams," JHEP 9801 (1998) 002, hep-th/9710116.

[10] N. C. Leung and C. Vafa, "Branes and Toric Geometry," Adv. Theor. Math. Phys. 2 (1998) 91-118, hep-th/9711013.

[11] B. Kol and J. Rahmfeld, "BPS Spectrum of 5 Dimensional Field Theories, $(p, q)$ Webs and Curve Counting," JHEP 9808 (1998) 006, hep-th/9801067. 
[12] Edward Witten, "Phase Transitions in M-Theory and F-Theory," Nucl. Phys. B471 (1996) 195-216, hep-th/9603150.

[13] N. Arkani-Hamed, A. G. Cohen and H. Georgi, "(De)Constructing Dimensions," Phys. Rev. Lett. 86 (2001) 4757, hep-th/0104005.

[14] Amer Iqbal, Vadim S. Kaplunovsky and Edoardo Di Napoli, "Quantum Deconstruction of $5 D S Q C D$ and its Moduli Space for General $n_{c}, n_{f}$ and $k_{\mathrm{cs}}$," in preparation.

[15] C. Csaki, J. Erlich, C. Grojean and G. Kribs, "4D Constructions of Supersymmetric Extra Dimensions and Gaugino Mediation," Phys. Rev. D65 (2002) 015003, hep-th/0106044.

[16] C. Csaki, J. Erlich, V. V. Khoze, E. Poppitz, Y. Shadmi and Y. Shirman, "Exact Results in 5D from Instantons and Deconstruction," Phys. Rev. D65 (2002) 085033, hep-th/0110188.

[17] Nathan Seiberg, "Exact Results on the Space of Vacua of Four Dimensional SUSY Gauge Theories," hep-th/9402044.

[18] C. Csaki, J. Erlich, D. Freedman and W. Skiba, "”N=1 Supersymmetric Product Group Theories in the Coulomb Phase," hep-th/9704067.

[19] Edward Witten, "Phases of $N=2$ Theories in Two Dimensions," Nucl. Phys. B403 (1993) 159-222, hep-th/9301042.

[20] J. M. Rodríguez, "Vacuum Structure of Supersymmetric SU $(N)^{K}$ Theories," Ph. D. Thesis (1997), UMI-98-25065-mc (microfiche).

[21] S. Chang and H. Georgi, "Quantum Modified Mooses" hep-th/0209038.

[22] W. Skiba and D. Smith, "Localized Fermions and Anomaly Inflow via Deconstruction," Phys. Lett. D65 (2002) 095, hep-ph/0201056.

[23] E. Di Napoli, unpublished work.

[24] David Morrison, "Geometric Aspects of Mirror Symmetry," math.AG/0007090. 